\title{
Analytical approximation methods for the stabilizing solution of the Hamilton-Jacobi equation
}

\author{
Noboru Sakamoto and Arjan J. van der Schaft
}

\begin{abstract}
In this paper, two methods for approximating the stabilizing solution of the Hamilton-Jacobi equation are proposed using symplectic geometry and a Hamiltonian perturbation technique as well as stable manifold theory. The first method uses the fact that the Hamiltonian lifted system of an integrable system is also integrable and regards the corresponding Hamiltonian system of the Hamilton-Jacobi equation as an integrable Hamiltonian system with a perturbation caused by control. The second method directly approximates the stable flow of the Hamiltonian systems using a modification of stable manifold theory. Both methods provide analytical approximations of the stable Lagrangian submanifold from which the stabilizing solution is derived. Two examples illustrate the effectiveness of the methods.
\end{abstract}

\section{INTRODUCTION}

When analyzing a control system or designing a feedback control, one often encounters certain types of equations that describe fundamental properties of the control problem at hand. It is the Riccati equation for linear systems and the Hamilton-Jacobi equation plays the same role in nonlinear systems. For example, an optimal feedback control can be derived from a solution of a Hamilton-Jacobi equation [25] and $H^{\infty}$ feedback controls are obtained by solving one or two Hamilton-Jacobi equations [5], [21], [38], [39]. Closely related to optimal control and $H^{\infty}$ control is the notion of dissipativity, which is characterized by a Hamilton-Jacobi inequality (see, e.g., [19], [42]). Some active areas of research in recent years are the factorization problem [6], [7] and the balanced realization problem [36], [15] and the solutions of these problems are again represented by Hamilton-Jacobi equations (or, inequalities). Contrary to the well-developed theory and computational tools for the Riccati equation, which are widely applied, the Hamilton-Jacobi equation is still an impediment to practical applications of nonlinear control theory.

In [27], [16], [30], [17] various series expansion techniques are proposed to obtain approximate solutions of the Hamilton-Jacobi equation. With these methods, one can calculate sub-optimal solutions using a few terms for simple nonlinearities. Although higher order approximations are possible to obtain for more complicated nonlinearities, their computations are often time-consuming and there is no guarantee that resulting controllers show better performance. Another approach is through successive approximation, where the Hamilton-Jacobi equation is reduced to a sequence of first order linear partial differential equations. The convergence of the algorithm is proven in [24]. In [9] an explicit technique to find approximate solutions to the sequence of partial differential equation is proposed using the Galerkin spectral method and in [41] the authors propose a modification of the successive approximation method and apply the convex optimization technique. The advantage of the Galerkin method is that it is applicable to a larger class of systems, while the disadvantages are that it is dependent on how well initial iterate is chosen and requires the calculation of $L^{2}$ inner products which can be significantly time-intensive for higher dimensional systems. The state-dependent Riccati equation approach is proposed in [20], [29] where a nonlinear function is rewritten in a linear-like representation. In this method, feedback control is given in a power series form and has a similar disadvantage to the

N. Sakamoto is with the Department of Aerospace Engineering, Nagoya University, Furo-cho, Chikusa-ku, Nagoya, 464-8603, Japan sakamoto@nuae.nagoya-u.ac.jp

A. J. van der Schaft is with the Institute for Mathematics and Computing Science, University of Groningen, 9700 AV Groningen, The Netherlands A.J.van.der.Schaft@math.rug.nl 
series expansion technique in that it is useful only for simple nonlinearities. A technique that employs open-loop controls and their interpolation is used in [28]. The drawback is that the interpolation of open-loop controls for each point in discretized state space is time-consuming and the computational cost grows exponentially with the state space dimension. A partially related research field to approximate solutions of the Hamilton-Jacobi equation is the theory of viscosity solutions. It deals with general Hamilton-Jacobi equations for which classical (differentiable) solutions do not exist. For introductions to viscosity solutions see, for instance, [8], [11], [13] and for an application to an $H^{\infty}$ control problem, see [37]. The finite-element and finite-difference methods are studied for obtaining viscosity solutions. They, however, require discretization of state space, which can be a significant disadvantage.

Another direction in the research for the Hamilton-Jacobi equation is to study the geometric structure and the properties of the equation itself and its exact solutions. The papers [38] and [39] give a sufficient condition for the existence of the stabilizing solution using symplectic geometry. In [35], the geometric structure of the Hamilton-Jacobi equation is studied showing the similarity and difference with the Riccati equation. See also [40] for the treatment of the Hamilton-Jacobi equation as well as recently developed techniques in nonlinear control theory such as the theory of port-Hamiltonian systems. In [32], the solution structure of a nonlinear optimal control problem is investigated using the inverted pendulum as an example.

In this paper, we focus on so-called stationary Hamilton-Jacobi equations which are related to, for example, infinite horizon optimal control problems and $H^{\infty}$ control problems, and attempt to develop methods to approximate the stabilizing solution of the Hamilton-Jacobi equation based on the geometric research in [38], [39] and [35]. The main object of the geometric research on the HamiltonJacobi equation is the associated Hamiltonian system. However, most approximation research papers mentioned above do not explicitly consider Hamiltonian systems, although it is well-known that the Hamiltonian matrix plays a crucial role in the calculation of the stabilizing solution for the Riccati equation. One of our purposes in this paper is to fill in this gap.

We will propose two analytical approximation methods for obtaining the stabilizing solution of the Hamilton-Jacobi equation. In the first method, we try to explore the possibility of using integrability conditions on the uncontrolled part of the system for controller design. Even when one can completely solve the equations of motion for a system with zero input, most nonlinear control techniques do not exploit the knowledge because once a feedback control is implemented, the system is not integrable anymore. However, within the geometric framework for the Hamilton-Jacobi equation, the effect of control can be considered as a Hamiltonian perturbation to the Hamiltonian system obtained by lifting the original equations of motion. Here, a crucial property is that if the equations of motion are integrable, then its lifted Hamiltonian system is also integrable. By using one of the Hamiltonian perturbation techniques (see, e.g., [4], [18]) we analyze the behaviors of the Hamiltonian systems with control effects and try to approximate the Lagrangian submanifold on which the Hamiltonian flow is asymptotically stable.

The second method in this paper takes the approach based on stable manifold theory (see, e.g., [10], [34]). Using the fact that the stable manifold of the associated Hamiltonian system is a Lagrangian submanifold and its generating function corresponds to the stabilizing solution, which is shown in [38], and modifying stable manifold theory, we analytically give the solution sequence that converges to the solution of the Hamiltonian system on the stable manifold. Thus, each element of the sequence approximates the Hamiltonian flow on the stable manifold and the feedback control constructed from each element may serve as an approximation of the desired feedback. It should be mentioned that computation methods of stable manifolds in dynamical systems are being developed and a comprehensive survey of the recent results in this area can be found in [22]. The proposed method in this paper, however, is different from the above numerical methods in that it gives analytical expressions of the approximated flows on stable manifolds, which may have considerable potential for control system 
designs that often lead to high dimensional Hamiltonian systems.

The organization of the paper is as follows. In $\S I I$, the theory of 1st-order partial differential equations is reviewed in the framework of symplectic geometry, stressing the one-to-one correspondence between solution and Lagrangian submanifold. In §III, a special type of solution, called the stabilizing solution, is introduced and the geometric theory for the Riccati equation is also reviewed. In the beginning of $\S \mathrm{IV}$ a key observation on integrability for Hamiltonian lifted systems is presented. We apply a Hamiltonian perturbation technique (reviewed in Appendix -A) for the system in which the Hamiltonian is decomposed into an integrable one and a perturbation Hamiltonian that is related to the influence of control. By assuming that the linearized Riccati equation at the origin has a stabilizing solution, we try to approximate the behaviors on the stable Lagrangian submanifold. In $\S \mathrm{V}$, an analytical approximation algorithm for the stable Lagrangian submanifold is proposed, using a modification of stable manifold theory. The proof of the main theorem in this section will be given in Appendix -B.

In $\S \mathrm{VI}-\mathrm{A}$, we address some computational issues. One of the eminent features of the approach taken in the paper is that we try to obtain not solutions of the Hamilton-Jacobi equation but submanifolds in the extended state space from which the solutions are produced by geometric integration (for example, Poincaré's lemma). However, only approximations of the submanifolds are obtained and the integrability condition does not hold anymore. We circumvent this difficulty, by obtaining derivatives of the solutions ( $\S \mathrm{VI}-\mathrm{A}$ ) or by using integral expressions of value functions in optimal control problems or storage functions in dissipative system theory (§VI-B). Also in $\S \mathrm{VI}-\mathrm{C}$, we touch on one of the advantages of our analytic approach, by showing that approximate solutions can be explicitly obtained as polynomial functions when the system under consideration has only polynomial nonlinearities. In $\S$ VII-A, we illustrate a numerical example showing the effectiveness of the proposed methods. Since this is a one-dimensional system, one can obtain the rigorous solution, which is convenient to see the accuracy and convergence of the methods. In §VII-B, we consider a two-dimensional problem, an optimal control of a nonlinear spring-mass system, in which the spring possesses nonlinear elasticity. Lastly, the Appendix includes the expositions for the variation of constants technique in Hamiltonian perturbation theory, proof of the main theorem in $\S \mathrm{V}$ and some formulas of the Jacobi elliptic functions used in $§$ VII-B.

\section{REVIEW OF THE THEORY OF 1ST-ORDER PARTIAL DIFFERENTIAL EQUATIONS}

In this section we outline, by using the symplectic geometric machinery, the essential parts of the theory of partial differential equations of the first order.

Let us consider a partial differential equation of the form

$$
F\left(x_{1}, \cdots, x_{n}, p_{1}, \cdots, p_{n}\right)=0,
$$

where $F$ is a $C^{\infty}$ function of $2 n$ variables, $x_{1}, \cdots, x_{n}$ are independent variables and $p_{1}=\partial z / \partial x_{1}, \cdots, p_{n}=$ $\partial z / \partial x_{n}$ with $z$ an unknown function. Since the Hamilton-Jacobi equation in nonlinear control theory does not explicitly depend on $z$, we did not include it in (PD). The contact geometry handles the time-varying case (see, e.g., [26]). Let $M$ be an $n$ dimensional space for $\left(x_{1}, \cdots, x_{n}\right)$. We regard the $2 n$ dimensional space for $(x, p)=\left(x_{1}, \cdots, x_{n}, p_{1}, \cdots, p_{n}\right)$ as the cotangent bundle $T^{*} M$ of $M$. $T^{*} M$ is a symplectic manifold with symplectic form $\theta=\sum_{i=1}^{n} d x_{i} \wedge d p_{i}$.

Let $\pi: T^{*} M \rightarrow M$ be the natural projection and $F^{-1}(0) \subset T^{*} M$ be a hypersurface defined by $F=0$. Define a submanifold

$$
\Lambda_{Z}=\left\{(x, p) \in T^{*} M \mid p_{i}=\partial z / \partial x_{i}(x), i=1, \cdots, n\right\}
$$

for a smooth function $z(x)$. Then, $z(x)$ is a solution of (PD) if and only if $\Lambda_{Z} \subset F^{-1}(0)$. Furthermore, $\left.\pi\right|_{\Lambda_{Z}}: \Lambda_{Z} \rightarrow M$ is a diffeomorphism and $\Lambda_{Z}$ is a Lagrangian submanifold because $\operatorname{dim} \Lambda_{Z}=n$ and

$$
\left.\theta\right|_{\Lambda_{Z}}=0 .
$$


Conversely, it is well-known (see, e.g. [1], [31]) that for a Lagrangian submanifold $\Lambda$ passing through $q \in T^{*} M$ on which $\left.\pi\right|_{\Lambda}: \Lambda \rightarrow M$ is a diffeomorphism, there exists a neighborhood $U$ of $q$ and a function $z(x)$ defined on $\pi(U)$ such that

$$
\Lambda \cap U=\left\{(x, p) \in U \mid p_{i}=\partial z / \partial x_{i}(x), i=1, \cdots, n\right\} .
$$

Therefore, finding a solution of $(\mathrm{PD})$ is equivalent to finding a Lagrangian submanifold $\Lambda \subset F^{-1}(0)$ on which $\left.\pi\right|_{\Lambda}: \Lambda \rightarrow M$ is a diffeomorphism.

Let $f_{1}=F$. To construct such a Lagrangian submanifold passing through $q \in T^{*} M$, and hence to obtain a solution defined on a neighborhood of $\pi(q)$, it is necessary and sufficient to find functions $f_{2}, \cdots, f_{n}$ on $T^{*} M$ with $d f_{1}(q) \wedge \cdots \wedge d f_{n}(q) \neq 0$ such that $\left\{f_{i}, f_{j}\right\}=0(i, j=1, \cdots, n)$, where $\{\cdot, \cdot\}$ is the canonical Poisson bracket, and

$$
\left|\frac{\partial\left(f_{1}, \cdots, f_{n}\right)}{\partial\left(p_{1}, \ldots, p_{n}\right)}\right|(q) \neq 0 .
$$

Using these functions, equations $f_{1}=0, f_{j}=$ constant, $j=2, \ldots, n$ define a Lagrangian submanifold $\Lambda \subset F^{-1}(0)$. Note that the condition (1) implies, by the implicit function theorem, that $\left.\pi\right|_{\Lambda}$ is a diffeomorphism on some neighborhood of $q$.

Since $\{F, \cdot\}$ is the Hamiltonian vector field $X_{F}$ with Hamiltonian $F$, the functions $f_{2}, \cdots, f_{n}$ above are first integrals of $X_{F}$. The ordinary differential equations that give the integral curve of $X_{F}$ are Hamilton's canonical equations

$$
\left\{\begin{array}{l}
\frac{d x_{i}}{d t}=\frac{\partial F}{\partial p_{i}} \\
\frac{d p_{i}}{d t}=-\frac{\partial F}{\partial x_{i}}
\end{array} \quad(i=1, \cdots, n)\right.
$$

and therefore, we seek $n-1$ commuting first integrals of (2) satisfying (1).

\section{The Stabilizing SOlution of the Hamilton-Jacobi EQUATion}

Let us consider the Hamilton-Jacobi equation in nonlinear control theory

$$
H(x, p)=p^{T} f(x)-\frac{1}{2} p^{T} R(x) p+q(x)=0,
$$

where $p_{1}=\partial V / \partial x_{1}, \cdots, p_{n}=\partial V / \partial x_{n}$ with $V(x)$ an unknown function, $f: M \rightarrow \mathbb{R}^{n}, R: M \rightarrow \mathbb{R}^{n \times n}$, $q: M \rightarrow \mathbb{R}$ are all $C^{\infty}$, and $R(x)$ is a symmetric matrix for all $x \in M$. We also assume that $f$ and $q$ satisfy $f(0)=0, q(0)=0$ and $\frac{\partial q}{\partial x}(0)=0$. In what follows, we write $f(x), q(x)$ as $f(x)=A x+O\left(|x|^{2}\right)$, $q(x)=\frac{1}{2} x^{T} Q x+O\left(|x|^{2}\right)$ where $A$ is an $n \times n$ real matrix and $Q \in \mathbb{R}^{n \times n}$ is a symmetric matrix.

The stabilizing solution of (HJ) is defined as follows.

Definition 1: A solution $V(x)$ of (HJ) is said to be the stabilizing solution if $p(0)=0$ and 0 is an asymptotically stable equilibrium of the vector field $f(x)-R(x) p(x)$, where $p(x)=(\partial V / \partial x)^{T}(x)$.

It will be important to understand the notion of the stabilizing solution in the framework of symplectic geometry described in the previous section. Suppose that we have the stabilizing solution $V(x)$ around the origin. Then, the Lagrangian submanifold corresponding to $V(x)$ is

$$
\Lambda_{V}=\{(x, p) \mid p=\partial V / \partial x(x)\} \subset T^{*} M
$$

$\Lambda_{V}$ is invariant under the Hamiltonian flow generated by

$$
\left\{\begin{array}{l}
\dot{x}=f(x)-R(x) p \\
\dot{p}=-\frac{\partial f}{\partial x}(x)^{T} p+\frac{\partial\left(p^{T} R(x) p\right)^{T}}{\partial x}-\frac{\partial q^{T}}{\partial x} .
\end{array}\right.
$$


To see this invariance, one needs to show that the second equation identically holds on $\Lambda_{V}$, which can be done by taking the derivative of (HJ) after replacing $p$ with $p(x)$. Note that the right-hand side in the second equation of (3) restricted to $\Lambda_{V}$ is $(\partial p / \partial x)(f(x)-R(x) p(x))$. The first equation is exactly the vector field in Definition 1. Therefore, any stabilizing solution is a generating function of the Lagrangian submanifold on which $\pi$ is a diffeomorphism and the Hamiltonian flow associated with $H(x, p)$ is asymptotically stable.

It is also useful to see the same picture for the Riccati equation;

$$
\text { (RIC) } \quad P A+A^{T} P-P R(0) P+Q=0,
$$

which is the linearization of (HJ). A symmetric matrix $P$ is said to be the stabilizing solution of (RIC) if it is a solution of (RIC) and $A-R(0) P$ is stable. The $2 n \times 2 n$ matrix

$$
\operatorname{Ham}=\left(\begin{array}{cc}
A & -R(0) \\
-Q & -A^{T}
\end{array}\right)
$$

is called the Hamiltonian matrix of (RIC) corresponding to the Hamiltonian vector field (3). A necessary and sufficient condition for the existence of the stabilizing solution [3], [33], [14], [23] is that (i) Ham has no eigenvalues on the imaginary axis, and (ii) the generalized eigenspace $\mathbb{E}_{-}$for $n$ stable eigenvalues satisfies the following complementarity condition;

$$
\mathbb{E}_{-} \oplus \operatorname{Im}\left(\begin{array}{l}
0 \\
I
\end{array}\right)=\mathbb{R}^{2 n}
$$

The condition (i) guarantees that the stable Lagrangian submanifold (subspace) does exist while (ii) corresponds to the diffeomorphism assumption of $\pi$ on the Lagrangian submanifold. More specifically, suppose that the assumptions (i), (ii) are satisfied, then the stabilizing solution $P$ exists to (RIC). Take the solution $S$ to the Lyapunov equation $(A-R(0) P) S+S(A-R(0) P)^{T}=R(0)$ and set

$$
T=\left(\begin{array}{cc}
I & S \\
P & P S+I
\end{array}\right)
$$

then it holds that

$$
\operatorname{Ham} T=T\left(\begin{array}{cc}
(A-R(0) P) & 0 \\
0 & -(A-R(0) P)^{T}
\end{array}\right) .
$$

A nonlinear (Hamilton-Jacobi) extension of (5) is found in [35].

We assume the following throughout the paper.

Assumption 1: The Riccati equation (RIC) satisfies conditions (i) and (ii), and thus has a stabilizing solution $P$ denoted by $\Gamma$.

\section{The HAMILTONiAn PERTURBATION APPROACH}

It is well-known that any system described by an ordinary differential equation can be represented as a Hamiltonian system by doubling the system dimension (Hamiltonian lifting). In [12] this technique is extended to control systems with inputs and outputs and is known to be effective for fundamental control problems such as factorization [7], [6] and model reduction problems [15]. We first give a useful observation on a Hamiltonian lifted system when the original system is integrable. Although it is simple, we did not find this observation in the literature.

Let the system $\dot{x}=f(x)$ be completely integrable and $u_{1}(x), \ldots, u_{n-1}(x)$ be first integrals. Consider its Hamiltonian lifted system

$$
\left\{\begin{array}{l}
\dot{x}=\frac{\partial H_{0}}{\partial p}=f(x) \\
\dot{p}=-\frac{\partial H_{0}}{\partial x}=-\frac{\partial f}{\partial x}(x)^{T} p
\end{array}\right.
$$


with Hamiltonian $H_{0}=p^{T} f(x)$. Let $v_{j}(x, p)=u_{j}(x)$ for $j=1, \ldots, n-1$ and $v_{n}(x, p)=H_{0}(x, p)$. Then,

$$
\begin{gathered}
\left\{v_{i}, v_{j}\right\}=\frac{\partial v_{i}}{\partial x} \frac{\partial v_{j}^{T}}{\partial p}-\frac{\partial v_{i}}{\partial p} \frac{\partial v_{j}^{T}}{\partial x}=0 \\
\quad \text { for } i, j=1, \ldots, n-1, \\
\left\{v_{i}, v_{n}\right\}=\frac{\partial v_{i}}{\partial x} f(x)-\frac{\partial v_{i}}{\partial p} \frac{\partial\left(p^{T} f(x)\right)^{T}}{\partial x}=0 \\
\quad \text { for } i=1, \ldots, n-1,
\end{gathered}
$$

which means that $v_{1}, \ldots, v_{n}$ are in involution. Therefore, the Hamiltonian system (6) is integrable in the sense of Liouville. This means that if one can obtain general solutions of the original system by quadrature, it is also possible for its lifted system.

One may realize that in the analysis of the Hamilton-Jacobi equation (HJ) Hamilton's canonical equations (3) contain the same terms as the Hamiltonian lifting (6) of the plant system. The purpose of this section is to show that one can exploit this property of Hamiltonian lifting for approximation of the stabilizing solution of (HJ).

Assumption 2: The system under control $\dot{x}=f(x)$ is completely integrable in the sense that there exist $n-1$ independent first integrals, and thus a solution $x=\Phi\left(t, x_{0}\right)$ for a general initial condition $x=x_{0}$ at $t=0$ is obtained.

Define the perturbation Hamiltonian by $H_{1}:=H-H_{0}=-\frac{1}{2} p^{T} R(x) p+q(x)$. The Hamiltonian $H_{1}$ is considered to represent the effect of the control inputs on the integrable system. We first solve the unperturbed Hamilton's canonical equations (6) determined by $H_{0}$ by means of the Hamilton-Jacobi theory. We take the Hamilton-Jacobi approach because it automatically produces new canonical variables. It is important to keep working with canonical variables so as not to cause secular terms in calculations, by which stability analysis may become unreliable (see, e.g., [18]). The Hamilton-Jacobi equation to solve (6) is

$$
H_{0}\left(x, \frac{\partial W}{\partial x}\right)+\frac{\partial W}{\partial t}=0
$$

Proposition 2: A complete solution of (7) is obtained as

$$
W(x, t, P)=\sum_{j=1}^{n} P_{j} \Phi_{j}(-t, x), \quad P_{j}^{\prime} \text { s: arbitrary constants, }
$$

where $\Phi(t, x)=\left(\Phi_{1}(t, x), \cdots, \Phi_{n}(t, x)\right)$ is the flow of $\dot{x}=f(x)$.

Proof: The characteristic equation for (7) is

$$
\left\{\begin{array}{l}
\frac{d x}{d s}=f(x(s)) \\
\frac{d t}{d s}=1
\end{array}\right.
$$

Since the general solution is $x(s)=\Phi\left(s, x_{0}\right), t(s)=s+s_{0}$, the $n$ independent integrals of the characteristic equation are $\Phi_{1}(-t, x), \ldots, \Phi_{n}(-t, x)$. To see this, we note that

$$
\begin{aligned}
\Phi(-t(s), x(s)) & =\Phi\left(-\left(s+s_{0}\right), \Phi\left(s, x_{0}\right)\right) \\
& \left.=\Phi\left(-s_{0}, x_{0}\right) \text { (independent of } s\right) .
\end{aligned}
$$

The general solution $W$ of the Hamilton-Jacobi equation (7) is an arbitrary function of the integrals $\Phi_{1}(-t, x), \ldots, \Phi_{n}(-t, x)$. We choose a linear combination of them with constants $P_{1}, \ldots, P_{n}$. 
From $W(x, t, P)$, by

$$
p_{j}=\frac{\partial W}{\partial x}, \quad X_{j}=\frac{\partial W}{\partial P_{j}}(\text { arbitrary constants }),
$$

a general solution of the lifted unperturbed system (6) is obtained as

$$
x_{j}(t)=\Phi_{j}(t, X), \quad p_{j}(t)=\sum_{k=1}^{n} P_{k} \frac{\partial \Phi}{\partial x_{j}}(-t, x)
$$

or,

$$
x(t, X)=\Phi(t, X), \quad p(t, X, P)=\frac{\partial \Phi}{\partial x}(-t, x)^{T} P .
$$

We note that the time-dependent transformation $(x, p) \rightarrow(X, P)$ is canonical. In the new coordinates the free motion (without control) is represented as

$$
\dot{X}=0, \quad \dot{P}=0 .
$$

With control, the perturbation Hamiltonian is in the coordinates $(X, P)$

$$
H_{1}(x, p)=H_{1}(x(t, X), p(t, X, P))=: \bar{H}_{1}(X, P, t),
$$

and $X, P$ obey

$$
\dot{X}_{j}=\frac{\partial \bar{H}_{1}}{\partial P_{j}}, \quad \dot{P}_{j}=-\frac{\partial \bar{H}_{1}}{\partial X_{j}}, \quad j=1, \ldots, n .
$$

We remark that until now no approximation has been made. If we plug the solution $X(t), P(t)$ of $(10)$ into (8) or (9), we get exact solutions of Hamilton's canonical equations (3) for the original control Hamilton-Jacobi equation (HJ) (see, Appendix -A). However, it is still difficult to solve (10) and we try to find an approximate solution of (10). Using the solution in Assumption 2, we have

$$
\begin{gathered}
x=\Phi(t, X)=e^{A t} X+O\left(|X|^{2}\right) \\
X=\Phi(-t, x)=e^{-A t} x+O\left(|x|^{2}\right) .
\end{gathered}
$$

Proposition 3: The linearized equation of (10) is

$$
\left\{\begin{array}{l}
\dot{\bar{X}}=-e^{-A t} R(0) e^{-A^{T} t} \bar{P} \\
\dot{\bar{P}}=-e^{A^{T} t} Q e^{A t} \bar{X}
\end{array}\right.
$$

Moreover, this can be explicitly solved as

$$
\left(\begin{array}{c}
\bar{X}\left(t, \bar{X}_{0}, \bar{P}_{0}\right) \\
\bar{P}\left(t, \bar{X}_{0}, \bar{P}_{0}\right)
\end{array}\right)=\left(\begin{array}{cc}
e^{A t} & 0 \\
0 & e^{-A^{T} t}
\end{array}\right)^{-1} \times \exp \left[t\left(\begin{array}{cc}
A & -R(0) \\
-Q & -A^{T}
\end{array}\right)\right]\left(\begin{array}{c}
\bar{X}_{0} \\
\bar{P}_{0}
\end{array}\right)
$$

Proof: The new Hamiltonian in $(X, P)$ coordinates is

$$
\begin{aligned}
\bar{H}_{1}= & -\frac{1}{2} P^{T} \frac{\partial \tilde{\Phi}}{\partial x}(t, x(t, X)) R(x(t, X)) \frac{\partial \tilde{\Phi}}{\partial x}(t, x(t, X))^{T} P \\
& +q(\Phi(t, X)) .
\end{aligned}
$$


Thus, we have

$$
\begin{aligned}
{\frac{\partial \bar{H}_{1}}{\partial P}=}^{T}= & \frac{\partial \tilde{\Phi}}{\partial x}(t, x(t, X)) R(x(t, X)) \frac{\partial \tilde{\Phi}}{\partial x}(t, x(t, X))^{T} P \\
\frac{\partial \bar{H}_{1}^{T}}{\partial X}= & \frac{\partial \Phi}{\partial X} \frac{\partial q}{\partial x}(\Phi(t, X))^{T} \\
& -\frac{\partial^{2} \tilde{\Phi}}{\partial x^{2}}\left(\frac{\partial \Phi}{\partial X}, R(x(t, X)) \frac{\partial \tilde{\Phi}}{\partial x} P\right)^{T} P \\
& -\left.\frac{1}{2} \frac{\partial\left(p^{T} R(x(t, X)) p\right)^{T}}{\partial X}\right|_{p=\frac{\partial \tilde{\Phi}}{\partial x}(t, x(t, X))^{T} P}
\end{aligned}
$$

where we have denoted $\tilde{\Phi}(t, x)=\Phi(-t, x)$ for simplicity and $\partial^{2} \tilde{\Phi} / \partial x^{2}(\cdot, \cdot)$ is a symmetric bilinear map. Noting (11) and (12), we collect first order terms of $X$ and $P$ in (10) to get (13). To solve (13), we set $\alpha=e^{A t} \bar{X}, \beta=e^{-A^{T} t} \bar{P}$. Then, we have

$$
\left(\begin{array}{c}
\dot{\alpha} \\
\dot{\beta}
\end{array}\right)=\left(\begin{array}{cc}
A & -R(0) \\
-Q & -A^{T}
\end{array}\right)\left(\begin{array}{l}
\alpha \\
\beta
\end{array}\right),
$$

from which the claim is derived using the inverse transformation.

Substituting the solution in Proposition 3 into (8) or (9), we obtain approximating flows of the Hamiltonian system (3). Now, we wish to select, among them, convergent flows to the origin, which are the approximations of the flows on the stable Lagrangian submanifold.

By Assumption 1, it follows that

$$
\exp \left[t\left(\begin{array}{cc}
A & -R(0) \\
-Q & -A^{T}
\end{array}\right)\right]\left(\begin{array}{l}
I \\
\Gamma
\end{array}\right)=\left(\begin{array}{l}
I \\
\Gamma
\end{array}\right) \exp [t(A-R(0) \Gamma)] .
$$

Therefore, if we take the initial conditions $\bar{X}_{0}$ and $\bar{P}_{0}$ satisfying $\bar{P}_{0}=\Gamma \bar{X}_{0}$ (stable Lagrangian subspace), then, we have

$$
\left(\begin{array}{l}
\bar{X} \\
\bar{P}
\end{array}\right)=\left(\begin{array}{cc}
e^{-A t} & 0 \\
0 & e^{A^{T} t}
\end{array}\right)\left(\begin{array}{l}
I \\
\Gamma
\end{array}\right) \exp [t(A-R(0) \Gamma)] \bar{X}_{0}
$$

Let us denote quantities in the left-hand side of the above equation as $\bar{X}\left(t, \bar{X}_{0}, \Gamma \bar{X}_{0}\right)$ and $\bar{P}\left(t, \bar{X}_{0}, \Gamma \bar{X}_{0}\right)$. Then, we have the following proposition.

Proposition 4: For sufficiently small $\left|\bar{X}_{0}\right|$,

$$
\begin{aligned}
x\left(t, \bar{X}_{0}\right) & =x\left(t, \bar{X}\left(t, \bar{X}_{0}, \Gamma \bar{X}_{0}\right)\right) \\
& =\Phi\left(t, e^{-A t} e^{(A-R(0) \Gamma) t} \bar{X}_{0}\right) \\
p\left(t, \bar{X}_{0}\right) & =p\left(t, \bar{X}\left(t, \bar{X}_{0}, \Gamma \bar{X}_{0}\right), \bar{P}\left(t, \bar{X}_{0}, \Gamma \bar{X}_{0}\right)\right) \\
& =\frac{\partial \Phi}{\partial x}\left(-t, x\left(t, \bar{X}_{0}\right)\right)^{T} e^{A^{T} t} \Gamma e^{(A-R(0) \Gamma) t} \bar{X}_{0}
\end{aligned}
$$

converge to the origin as $t \rightarrow \infty$.

Proof: This can be verified from (11), (12) and the fact that $A-R(0) \Gamma$ is an asymptotically stable matrix.

From Proposition 4, we think of (14) as approximate behaviors on the stable Lagrangian submanifold, and thus, (14) can be regarded as parameterized approximations of the Lagrangian submanifold. 
Summarizing, we propose the following method to approximate the stable Lagrangian submanifold and the stabilizing solution.

Procedure 1: Solve the uncontrolled system equation $\dot{x}=f(x)$. Form a general solution (8) or (9) of (6) using the solution $\Phi\left(t, x_{0}\right)$ of $\dot{x}=f(x)$. Find the stabilizing solution $P=\Gamma$ of (RIC) in Assumption 1. Then,

$$
\Lambda_{t}=\left\{(x, p) \mid p=\frac{\partial \Phi}{\partial x}(-t, x)^{T} e^{A^{T} t} \Gamma e^{A t} \Phi(-t, x)\right\}
$$

is a family of approximations of the stable Lagrangian submanifold. That is, $\frac{\partial \Phi}{\partial x}(-t, x)^{T} e^{A^{T} t} \Gamma e^{A t} \Phi(-t, x)$ is an approximation of the derivative $\partial V / \partial x$ of the stabilizing solution.

Proof: By eliminating $\bar{X}_{0}$ in (14), one can derive (15).

Remark IV.1: The set $\Lambda_{t}$ in (15) includes the linearized solution $p=\Gamma x$ for $t=0$. Also, it can be seen that for sufficiently small $|x|$, each surface in (15) is tangent to $p=\Gamma x$, from which one can expect that the performance of the feedback control using (15) is better than that of linear control using $P=\Gamma$ of (RIC). For a practical method of determining the value of $t$, see $\S$ VI-D.

\section{THE STABlE MANiFOLD THEORY APPROACH}

A. Approximation of stable manifolds

We consider the following system

$$
\left\{\begin{array}{l}
\dot{x}=F x+f(t, x, y) \\
\dot{y}=-F^{T} y+g(t, x, y) .
\end{array}\right.
$$

Assumption 3: $F$ is an asymptotically stable $n \times n$ real matrix and it holds that $\left\|e^{F t}\right\| \leqslant a e^{-b t}$, $t \geqslant 0$ for some constants $a>0$ and $b>0$.

Assumption 4: $f, g: \mathbb{R} \times \mathbb{R}^{n} \times \mathbb{R}^{n} \rightarrow \mathbb{R}^{n}$ are continuous and satisfy the following.

i) For all $t \in \mathbb{R},|x|+|y|<l$ and $\left|x^{\prime}\right|+\left|y^{\prime}\right|<l$,

$$
\left|f(t, x, y)-f\left(t, x^{\prime}, y^{\prime}\right)\right| \leqslant \delta_{1}(l)\left(\left|x-x^{\prime}\right|+\left|y-y^{\prime}\right|\right) .
$$

ii) For all $t \in \mathbb{R},|x|+|y|<l$ and $\left|x^{\prime}\right|+\left|y^{\prime}\right|<l$,

$$
\left|g(t, x, y)-g\left(t, x^{\prime}, y^{\prime}\right)\right| \leqslant \delta_{2}(l)\left(\left|x-x^{\prime}\right|+\left|y-y^{\prime}\right|\right)
$$

where $\delta_{j}:[0, \infty) \rightarrow[0, \infty), j=1,2$ are continuous and monotonically increasing on $\left[0, L_{j}\right]$ for some constants $L_{1}, L_{2}>0$.

Furthermore, there exist constants $M_{1}, M_{2}>0$ such that $\delta_{j}(l) \leqslant M_{j} l$ holds on $\left[0, L_{j}\right]$ for $j=1,2$.

Let us define the sequences $\left\{x_{k}(t, \xi)\right\}$ and $\left\{y_{k}(t, \xi)\right\}$ by

$$
\left\{\begin{array}{l}
x_{k+1}=e^{F t} \xi+\int_{0}^{t} e^{F(t-s)} f\left(s, x_{k}(s), y_{k}(s)\right) d s \\
y_{k+1}=-\int_{t}^{\infty} e^{-F^{T}(t-s)} g\left(s, x_{k}(s), y_{k}(s)\right) d s
\end{array}\right.
$$

for $k=0,1,2, \ldots$, and

$$
\left\{\begin{array}{l}
x_{0}=e^{F t} \xi \\
y_{0}=0
\end{array}\right.
$$

with arbitrary $\xi \in \mathbb{R}^{n}$. 
The following theorem states that the sequences $\left\{x_{k}(t, \xi)\right\},\left\{y_{k}(t, \xi)\right\}$ are the approximating solutions to the exact solution of (16) on the stable manifold with the property that each element of the sequences is convergent to the origin.

Theorem 5: Under Assumptions 3 and $4, x_{k}(t, \xi)$ and $y_{k}(t, \xi)$ are convergent to zero for sufficiently small $|\xi|$, that is, $x_{k}(t, \xi), y_{k}(t, \xi) \rightarrow 0$ as $t \rightarrow \infty$ for all $k=0,1,2, \ldots$ Furthermore, $x_{k}(t, \xi)$ and $y_{k}(t, \xi)$ are uniformly convergent to a solution of $(16)$ on $[0, \infty)$ as $k \rightarrow \infty$. Let $x(t, \xi)$ and $y(t, \xi)$ be the limits of $x_{k}(t, \xi)$ and $y_{k}(t, \xi)$, respectively. Then, $x(t, \xi), y(t, \xi)$ are the solution on the stable manifold of (16), that is, $x(t, \xi), y(t, \xi) \rightarrow 0$ as $t \rightarrow \infty$.

Proof: See Appendix -B.

\section{B. The approximation algorithm}

Extracting the linear part in $(\mathrm{HJ}),(3)$ can be written as

$$
\left(\begin{array}{c}
\dot{x} \\
\dot{p}
\end{array}\right)=\left(\begin{array}{cc}
A & -R(0) \\
-Q & -A^{T}
\end{array}\right)\left(\begin{array}{l}
x \\
p
\end{array}\right)+\text { higher order terms. }
$$

Using the linear coordinate transformation

$$
\left(\begin{array}{l}
x^{\prime} \\
p^{\prime}
\end{array}\right)=T\left(\begin{array}{l}
x \\
p
\end{array}\right)
$$

where $T$ is defined in (4), the linear part of (19) is diagonalized as

$$
\left(\begin{array}{c}
\dot{x}^{\prime} \\
\dot{p}^{\prime}
\end{array}\right)=\left(\begin{array}{cc}
A-R(0) \Gamma & 0 \\
0 & -(A-R(0) \Gamma)^{T}
\end{array}\right)\left(\begin{array}{l}
x^{\prime} \\
p^{\prime}
\end{array}\right)+\text { higher order terms. }
$$

For (21), Assumption 1 implies Assumption 3 and Assumption 4 is satisfied if $f, R$ and $q$ in (HJ) are sufficiently smooth. Thus, we propose the following procedure for parametrized approximation of the stable Lagrangian submanifold.

Procedure 2: (i) Construct the sequences (17) for (21) and obtain the sequences $\left\{x_{k}(t, \xi)\right\}$, $\left\{p_{k}(t, \xi)\right\}$ in the original coordinates using $(20)$.

(ii) Take a small $r>0$ so as for the convergence of (17) to be guaranteed for $\xi$ in

$$
S_{r}=\left\{\left(\xi_{1}, \ldots, \xi_{n}\right) \mid \sum_{j=1}^{n} \xi_{j}^{2}=r^{2}\right\}
$$

Then,

$$
\Lambda_{k}=\left\{\left(x_{k}(t, \xi), p_{k}(t, \xi)\right) \mid t \in \mathbb{R}, \xi \in S_{r}\right\}
$$

is an approximation of the stable Lagrangian submanifold and $\Lambda_{k} \rightarrow \Lambda$ as $k \rightarrow \infty$, where $\Lambda$ is the stable Lagrangian submanifold whose existence is assured by Assumption 1 and the results in [38].

Remark V.1: Procedure 1 applies, compared to Procedure 2, to a smaller class of systems and does not provide a sequential method. However, since a nonlinearity is fully taken into account in Procedure 1, it gives a qualitatively good approximation with a large valid range (see, Example VIIA). Nevertheless, one may wish to obtain better approximations in the Hamiltonian perturbation approach. To this end, we have included the dependence on $t$ in (16), so as to be able to apply Procedure 2 to (10). More specifically, one applies the transformation $\alpha=e^{A t} X, \beta=e^{-A^{T} t} P$ as in the proof of Proposition 3 to get

$$
\left(\begin{array}{c}
\dot{\alpha} \\
\dot{\beta}
\end{array}\right)=\left(\begin{array}{cc}
A & -R(0) \\
-Q & -A^{T}
\end{array}\right)\left(\begin{array}{l}
\alpha \\
\beta
\end{array}\right)+\text { higher order terms, }
$$

where the higher order terms above are dependent on $t$ since $\bar{H}_{1}$ is time-dependent. Thus, Procedure 2 can be employed while the first approximation corresponds to the one in Procedure 1. 


\section{Vi. Computational issues}

One of the unique features of the approach taken in this paper is to obtain a certain $n$-dimensional surface (Lagrangian submanifold) in $2 n$-dimensional space, which is a graph of the derivative of the solution, and from the integrability property of the surface, the existence of the solution is guaranteed. For the purpose of the control system design, however, the actual computation of the solution and/or its derivative is necessary.

\section{A. Computation of $\partial V / \partial x$}

In $\S \mathrm{IV}$, the computation for $\partial V / \partial x$ is possible by eliminating $\bar{X}_{0}$ in (14). To obtain an approximation of $\partial V / \partial x$ in (22), suppose that $S_{r}$ is parametrized with $\left(\eta_{1}, \ldots, \eta_{n-1}\right)$. If one eliminates $t, \eta_{1}, \ldots, \eta_{n-1}$ from $2 n$ equations $x=x_{k}\left(t, \xi\left(\eta_{1}, \ldots, \eta_{n-1}\right)\right), p=p_{k}\left(t, \xi\left(\eta_{1}, \ldots, \eta_{n-1}\right)\right)$, the relation $p=\pi_{k}(x)$ is obtained and $\pi_{k}(x)$ will serve as an approximation of $\partial V / \partial x$. The elimination of variables in this case is, however, not easy to carry out in practice. An effective use of software is required for this purpose. In $\S \mathrm{VII}$, we interpolate the values of $p_{k}$ for sample points of $x_{k}$ to get the function $\pi_{k}(x)$ using MATLAB ${ }^{\circledR}$ commands such as griddatan and interpn.

\section{B. Computation of $V$}

The approximations of the stable Lagrangian submanifold $\Lambda_{t}$ in Procedure 1 and $\Lambda_{k}$ in Procedure 2 do not satisfy the integrability condition. Therefore, it is difficult to get an approximation of the generating function for the Lagrangian submanifold in a geometric manner. However, since we have analytical expressions of the approximations, we can write down approximations of the generating function as described below.

\section{B.1 Optimal control problem}

Let us consider the following optimal control problem:

$$
\dot{x}=f(x)+g(x) u, \quad f(0)=0
$$

with the cost function

$$
J=\int_{0}^{\infty} L(x(t), u(t)) d t
$$

where $g(x)$ is a smooth $n \times m$ matrix-valued function and $L$ takes the form of, for example, $L=$ $\left(h(x)^{T} h(x)+u^{T} u\right) / 2$ with smooth $h(x) \in \mathbb{R}^{r}, h(0)=0$. The optimal feedback control is given by

$$
u=-g(x)^{T} \frac{\partial V}{\partial x}(x)^{T}
$$

where $V(x)$ is the stabilizing solution of the corresponding Hamilton-Jacobi equation

$$
\frac{\partial V}{\partial x} f(x)-\frac{1}{2} \frac{\partial V}{\partial x} g(x) g(x)^{T}\left(\frac{\partial V}{\partial x}\right)^{T}+\frac{1}{2} h^{T}(x) h(x)=0 .
$$

By Procedure 2, the $k$-th approximation of the Lagrangian submanifold is parametrized as $\Lambda_{k}$ in $(22)$, and the $k$-th approximation of the optimal feedback can be described with $t$ and $\xi$ as

$$
u_{k}(t, \xi)=-g\left(x_{k}(t, \xi)\right)^{T} p_{k}(t, \xi) .
$$

Since the generating function is the minimum value of $J$ for each $\xi$, its approximation can be written as

$$
V_{k}(\xi)=\int_{0}^{\infty} L\left(x_{k}(t, \xi), u_{k}(t, \xi)\right) d t .
$$


The same computation is possible in the Hamiltonian perturbation approach, when $\dot{x}=f(x)$ is integrable, by using (14).

\section{B.2 $H^{\infty}$ control problem}

Let us consider the nonlinear system (23) with disturbances $w \in \mathbb{R}^{q}$

$$
\dot{x}=f(x)+g(x) u+l(x) w,
$$

where $l(x)$ is a smooth $n \times q$ matrix function. The state feedback $H^{\infty}$ control problem is to find a feedback control law $u=u(x)$ such that the closed loop system is asymptotically stable and has the $L^{2}$-gain (see, e.g., [39] for definition) from $w$ to $y=h(x)$ less than or equal to $\gamma>0$.

A sufficient condition for the solvability of the $H^{\infty}$ problem is that there exists a stabilizing solution $V(x) \geqslant 0$ to

$$
\frac{\partial V}{\partial x} f(x)+\frac{1}{2} \frac{\partial V}{\partial x}\left[\frac{1}{\gamma^{2}} l(x) l(x)^{T}-g(x) g(x)^{T}\right] \cdot\left(\frac{\partial V}{\partial x}\right)^{T}+\frac{1}{2} h^{T}(x) h(x)=0,
$$

and the feedback law is given by

$$
u^{*}=-g(x)^{T}\left(\frac{\partial V}{\partial x}\right)^{T} .
$$

Procedure 2 can be applied if the linearized $H^{\infty}$ problem is solvable and we can construct $k$-th approximation $\Lambda_{k}$ as in (22). From Pontryagin's minimum principle, one can show that

$$
\begin{aligned}
V(x) & =-\min _{\substack{w \in L^{2} \\
x(0)=x \\
x(\infty)=0}} \frac{1}{2} \int_{0}^{\infty} \gamma^{2}|w(t)|^{2}-|y(t)|^{2} d t \\
& =-\frac{1}{2} \int_{0}^{\infty} \gamma^{2} w^{*}(x(t))^{T} w^{*}(x(t))-h(x(t))^{T} h(x(t)) d t,
\end{aligned}
$$

where

$$
w^{*}=\frac{1}{\gamma^{2}} l(x)^{T} p(x)
$$

is the worst disturbance, $p(x)=(\partial V / \partial x)^{T}$ and $x(t)$ is the solution of the system $\dot{x}=f(x)+g(x) u^{*}(x)+$ $l(x) w^{*}(x)$. Then, $k$-th approximation for $V$ is given, by replacing $x, p(x)$ with $x_{k}(t, \xi), p_{k}(t, \xi)$ respectively, as

$$
V_{k}(\xi)=\frac{1}{2} \int_{0}^{\infty} \gamma^{2} w_{k}^{T}(t, \xi) w_{k}(t, \xi)-h\left(x_{k}(t, \xi)\right)^{T} h\left(x_{k}(t, \xi)\right) d t
$$

where $w_{k}(t, \xi)=\frac{1}{\gamma^{2}} l\left(x_{k}(t, \xi)\right)^{T} p_{k}(t, \xi)$.

When one designs a feedback control law and only the derivative of the solution of (HJ) is necessary, we recommend to employ the method in $\S \mathrm{VI}-\mathrm{A}$. This is because the operations in (24) or (25) have no effect of approximating the exact solution and the derivatives of these functions may be less accurate than those obtained by the method in $\S$ VI-A for the same $k$. The accuracy can be increased by taking larger $k$ and the two kinds of approximate derivatives coincide when $k \rightarrow \infty$.

\section{A special case-polynomial nonlinearities}

When (HJ) contains only polynomial nonlinearities, computations for $\partial V / \partial x$ and $V$ are carried out with elementary functions in the stable manifold theory approach in $\S \mathrm{V}$. In this case, the higher order terms in (19) are second or higher order polynomials, and so are $f, g$ in (17). 
The first approximations, corresponding to the linear solution, are $x_{0}=e^{F t}, y_{0}=0$ consisting of exponential and trigonometric functions. They are substituted in (17) yielding also exponential and trigonometric functions since $f$ and $g$ are polynomial. The second approximations are obtained by integrating them after multiplication of the matrix exponential $e^{F t}$, thus consisting of exponential and trigonometric functions. This continues for all $k$. Moreover, the integrands in (24) and (25) are also polynomials of $x$ and $p$, and therefore, $V_{k}(\xi)$ 's are obtained as polynomial functions of $\xi$.

\section{Determination of parameters and the radius of convergence}

In the perturbation methods, one needs to determine the value of $t$ so that (15) gives a good approximation of $\partial V / \partial x$ in some sense. We propose a practical method of doing that using the value of Hamiltonian $H$. If $V(x)$ is a solution of $(\mathrm{HJ}), H(x, \partial V / \partial x)=0$. Thus, if $p=p(x ; t)$ is an approximation of $\partial V / \partial x$ with parameter $t$, it may be reasonable to chose $t$ so as $\int|H(x, p(x ; t))| d x$ to be minimized.

In the stable manifold approach, on the other hand, one needs to estimate the radius of convergence $|\xi|$ of the sequence (17). Since obtaining a theoretical estimation for such a convergence domain is quite difficult and it tends to be conservative, we propose a practical method using the values of $H$ for each iteration. If $|\xi|$ is such that the iteration (22) is convergent, then $H\left(x_{k}(0, \xi), p_{k}(0, \xi)\right)$ is small. However, as $|\xi|$ grows beyond the radius of convergence, the value may rapidly increase. By looking at this change of $H$ for each $k$, one can reasonably estimate the radius of convergence.

The radius of convergence in the stable manifold approach is generally small, meaning that the resultant solution surface (22) is small around the origin if only positive $t$ is used. To enlarge the domain of the solution, one may try to use negative $t$. This, however, is an unstable direction of the flows and creates a divergent effect. We employ a similar idea to the above to see how much negative $t$ can be substituted in (22). For a fixed value of $\xi$, where $|\xi|$ is the radius of convergence, calculate $H\left(x_{k}(t, \xi), p_{k}(t, \xi)\right)$. Then, for negative $t$, as long as $\left(x_{k}(t, \xi), p_{k}(t, \xi)\right)$ stays near the exact solution (Lagrangian submanifold), the value is small. By looking at the growth of this value with respect to $t$, one can see how much negative $t$ can be substituted. If the domain thus obtained is not large enough, raise $k$ and use smaller $t$.

All of these methods are effectively applied using analytical expressions. We will demonstrate them in the next example.

\section{EXAMPLES}

\section{A. A numerical example}

Let us consider the 1-dimensional nonlinear optimal control problem;

$$
\begin{aligned}
& \dot{x}=x-x^{3}+u \\
& J=\int_{0}^{\infty} \frac{q}{2} x^{2}+\frac{r}{2} u^{2} d t .
\end{aligned}
$$

The Hamilton-Jacobi equation for this problem is

$$
H=p\left(x-x^{3}\right)-\frac{1}{2 r} p^{2}+\frac{q}{2} x^{2}=0
$$

and Hamilton's canonical equations are

$$
\left\{\begin{array}{l}
\dot{x}=x-x^{3}-\frac{1}{r} p \\
\dot{p}=-\left(1-3 x^{2}\right) p-q x .
\end{array}\right.
$$


A.1 The Hamiltonian perturbation method

The Hamiltonian $H$ is split into the integrable and perturbation parts;

$$
H_{0}=p\left(x-x^{3}\right), \quad H_{1}=-\frac{1}{2 r} p^{2}+\frac{q}{2} x^{2} .
$$

The solution of (26) with the initial condition $x=X$ at $t=0$ without control is obtained from

$$
\frac{x^{2}}{1-x^{2}}=\frac{X^{2}}{1-X^{2}} e^{2 t}
$$

and is denoted as $x=\Phi(t, X)$. The solution of the canonical equations for $H_{0}$ corresponding to (8) is

$$
x=\Phi(t, X), \quad p=\frac{\partial \Phi}{\partial x}(-t, x) P=\frac{X^{3} e^{2 t}}{x^{3}} P,
$$

where $P$ is an arbitrary constant and the last equation is derived from (29).

Based on the linearization of (26), the linearized canonical equations for perturbation that correspond to $(10)$ are

$$
\left\{\begin{array}{l}
\dot{\bar{X}}=-\frac{1}{r} e^{-2 t} \bar{P} \\
\dot{\bar{P}}=-q e^{2 t} \bar{X}
\end{array}\right.
$$

The solution of the above equations for the initial condition in the stable Lagrangian subspace of the linearized Riccati equation of (27) is

$$
\left\{\begin{array}{l}
\bar{X}\left(t, \bar{X}_{0}, \Gamma \bar{X}_{0}\right)=e^{-(1+\lambda) t} \bar{X}_{0} \\
\bar{P}\left(t, \bar{X}_{0}, \Gamma \bar{X}_{0}\right)=e^{(1-\lambda) t} \Gamma \bar{X}_{0}
\end{array}\right.
$$

where $\Gamma=r+\sqrt{r^{2}+q r}$ is the stabilizing solution of the Riccati equation and $-\lambda=-\sqrt{1+q / r}$ is the closed loop matrix (eigenvalue). The family of approximations of the stable Lagarangian submanifold in Procedure 1 is

$$
\Lambda_{t}: p=\frac{\Gamma x}{\left(\left(e^{-2 t}-1\right) x^{2}+1\right)^{2}} .
$$

The feedback function with $t=-0.2$ is shown in Fig. 1. Also, we showed the result by the Taylor series expansion of order $n=6$ for the sake of comparison. Since the integrable nonlinearity is fully taken into account in this approach, the feedback function is better approximated in the region further from the origin.

The value $t=-0.2$ was chosen by the method in $\S$ VI-D. Fig. 2 shows that $\int_{0}^{5}|H(x, p(x ; t))| d x$ takes the minimum value at $t=-0.2$.

\section{A.2 The stable manifold approximation method}

The coordinate transformation that diagonalizes the linear part of $(28)$ is

$$
\begin{gathered}
\left(\begin{array}{l}
x \\
p
\end{array}\right)=T\left(\begin{array}{l}
x^{\prime} \\
p^{\prime}
\end{array}\right), \\
T=\left(\begin{array}{cc}
1 \\
r+\sqrt{r^{2}+q r} & -(1+\sqrt{1+q / r})
\end{array}\right) .
\end{gathered}
$$

The equations in the new coordinates are

$$
\left(\begin{array}{c}
\dot{x}^{\prime} \\
\dot{p}^{\prime}
\end{array}\right)=\left(\begin{array}{c}
-\sqrt{1+q / r} x^{\prime} \\
\sqrt{1+q / r} p^{\prime}
\end{array}\right)+\left(\begin{array}{c}
-x\left(x^{\prime}, p^{\prime}\right)^{3} \\
3 x\left(x^{\prime}, p^{\prime}\right)^{2} p\left(x^{\prime}, p^{\prime}\right)
\end{array}\right),
$$




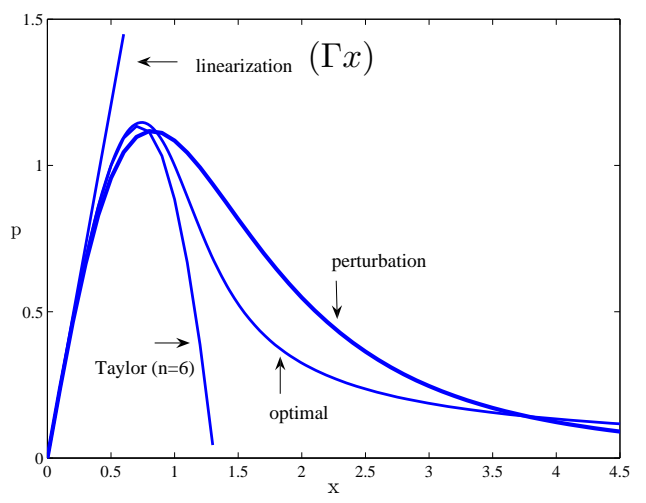

Fig. 1. Perturbation and Taylor expansion solutions



Fig. 2. Integration of error

where

$$
\begin{aligned}
& x\left(x^{\prime}, p^{\prime}\right)=x^{\prime}-(1+\sqrt{1+q / r}) p^{\prime}, \\
& p\left(x^{\prime}, p^{\prime}\right)=\left(r+\sqrt{r^{2}+q r}\right) x^{\prime}+q p^{\prime} .
\end{aligned}
$$

We construct the sequences (17) with

$$
\begin{gathered}
f\left(x^{\prime}, p^{\prime}\right)=-x\left(x^{\prime}, p^{\prime}\right)^{3}, \\
g\left(x^{\prime}, p^{\prime}\right)=3 x\left(x^{\prime}, p^{\prime}\right)^{2} p\left(x^{\prime}, p^{\prime}\right),
\end{gathered}
$$

and $q=1, r=1$. From $x_{k}(t, \xi)$ and $p_{k}(t, \xi)$, the relation of $x_{k}$ and $p_{k}$ is obtained by eliminating $t$, which will be denoted as $p=\pi_{k}(x)$. We note that $\pi_{k}(x)$ depends on $\xi$. The approximated feedback functions are $u=-(1 / r) \pi_{k}(x)=-\pi_{k}(x)$.

Figures 3-5 show the results of calculation for $\pi_{k}(x)$. To guarantee the convergence of solution sequence (17), $|\xi|$ has to be small enough (Theorem 5). If $|\xi|$ is too large, the sequence is not convergent (compare Fig. 3 and Fig. 5). We have estimated the radius of convergence using the method in $\S$ VI-D. From Fig. 6, one can see that $|\xi| \leqslant 0.42$ may be a reasonable estimation.

If $|\xi|$ is small and only positive $t$ is used in $x_{k}(t, \xi)$ and $p_{k}(t, \xi)$, then the resulting trace in the $x-p$ plane is short, hence, the function $\pi_{k}(x)$ is defined in a small set around the origin. Therefore, we substitute negative values in $t$ to extend the trace toward the opposite direction. This, however, creates a divergent effect on the sequence and this effect becomes smaller as $k$ increases (see, Fig. 4). We employed the approach in §VI-D to see how much negative time can be used in (22) to create a 
larger domain of $\pi_{k}(x)$. From Fig. 7, one can see that the domain of $\pi_{2}(x)$ may be enlarged up to $t=-0.5$. If this domain is not large enough, one should raise $k$ and substitute smaller $t$ (see, Fig. 3 and Fig. 4).

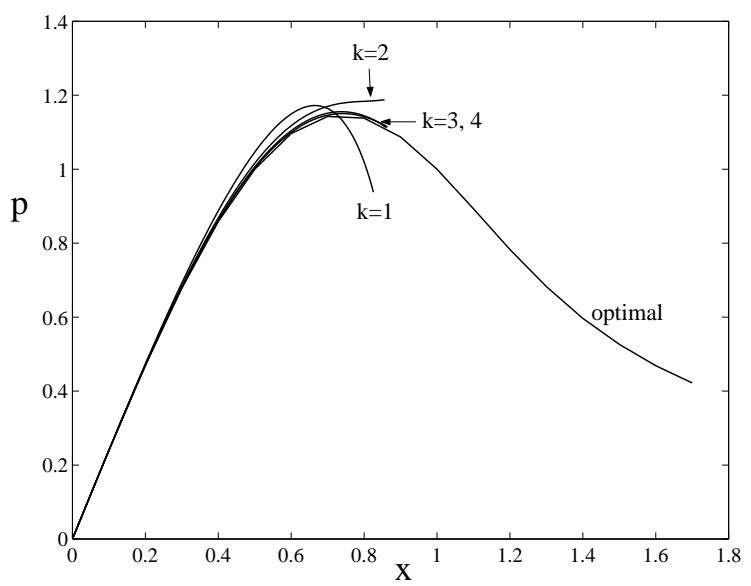

Fig. 3. $\xi=0.42$ and extended to the negative time -0.5

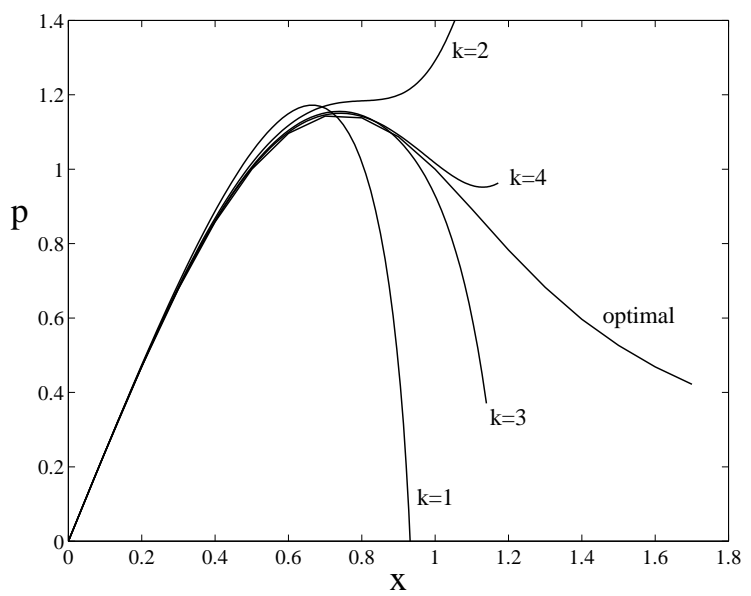

Fig. 4. $\xi=0.42$ and extended to the negative time -0.8

\section{B. Optimal control of a nonlinear spring-mass system}

In this example, let us consider an optimal control problem for a spring-mass system with input $u$ :

$$
\begin{gathered}
m \ddot{x}+\kappa x+\varepsilon x^{3}=u \\
J=\int_{0}^{\infty}\left(x^{2}+\dot{x}^{2}\right) / 2+u^{2} d t
\end{gathered}
$$

where, $m$ is the mass of an object attached to the spring, $x$ is the displacement of the object from rest (at rest, $x=0$; the spring generates no force), $\kappa$ and $\varepsilon$ are the linear and nonlinear spring constants, respectively. Hereafter, we set $m=1, \kappa=1$ for the sake of simplicity. The Hamilton-Jacobi equation for this problem is

$$
H=\dot{x} p_{1}+\left(-x-\varepsilon x^{3}\right) p_{2}-\frac{1}{2} p_{2}{ }^{2}+\frac{1}{2} x^{2}+\frac{1}{2} \dot{x}^{2}=0 .
$$




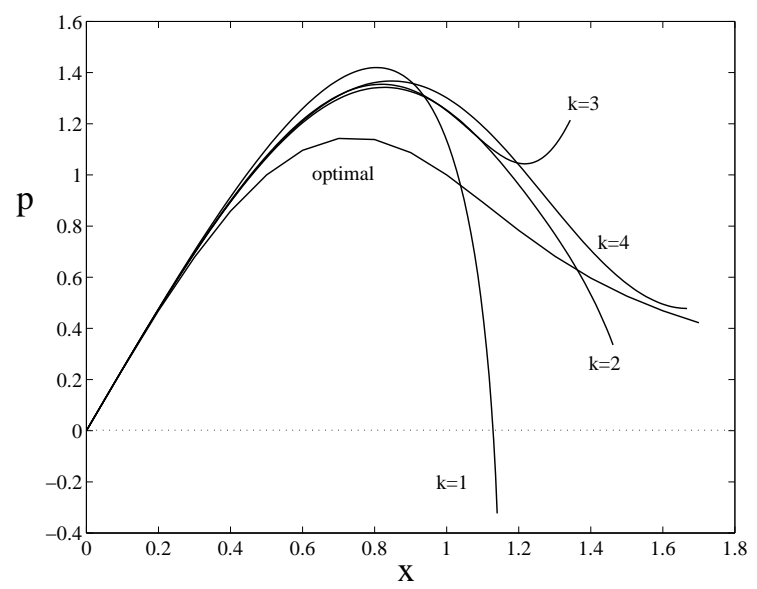

Fig. 5. $\quad \xi=0.6$ and extended to the negative time -0.5

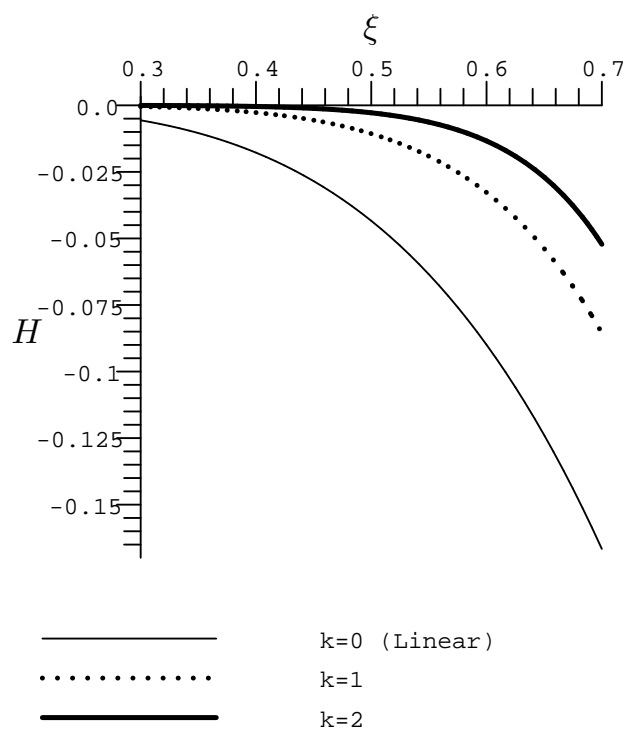

Fig. 6. Errors $H\left(x_{k}(0, \xi), p_{k}(0, \xi)\right)$

B.1 The perturbation approach

Equation (30) with initial condition $x_{0}, \dot{x}_{0}$ and no input $(u=0)$ is integrated as follows

$$
x=a \operatorname{cn}\left(\sqrt{1+\varepsilon a^{2}}\left(t_{0}-t\right), k\right),
$$

where, $a=\sqrt{\frac{\sqrt{1+4 \varepsilon E}-1}{\varepsilon}}$ with $E=\frac{1}{2} x_{0}^{2}+\frac{1}{4} \varepsilon x_{0}^{4}+\frac{1}{2} \dot{x}_{0}^{2}$, cn is the Jacobi elliptic function, and $k=\sqrt{\frac{\varepsilon a^{2}}{2\left(1+\varepsilon a^{2}\right)}}$ is the elliptic modulus. $t_{0}$ is a constant of integration and can be expressed using $a$ and $k$ as follows

$$
t_{0}=\frac{1}{\sqrt{1+\varepsilon a^{2}}} \mathrm{cn}^{-1}\left(\frac{x_{0}}{a}, k\right) .
$$

Note that $a, k, t_{0}$ are functions of $x_{0}, \dot{x}_{0}$. To express $x$ as a function of $t, x_{0}$ and $\dot{x}_{0}$, we substitute $a\left(x_{0}, \dot{x}_{0}\right), k\left(x_{0}, \dot{x}_{0}\right), t_{0}\left(x_{0}, \dot{x}_{0}\right)$ into (32) and use the addition formulas of the Jacobi elliptic functions (see, Appendix -C). Thus, $\Phi(t, x)$ in $§ I V$ is given as

$$
\Phi\left(t, x_{0}, \dot{x}_{0}\right)=\left(\begin{array}{c}
x\left(t, x_{0}, \dot{x}_{0}\right) \\
\frac{d}{d t} x\left(t, x_{0}, \dot{x}_{0}\right)
\end{array}\right) .
$$




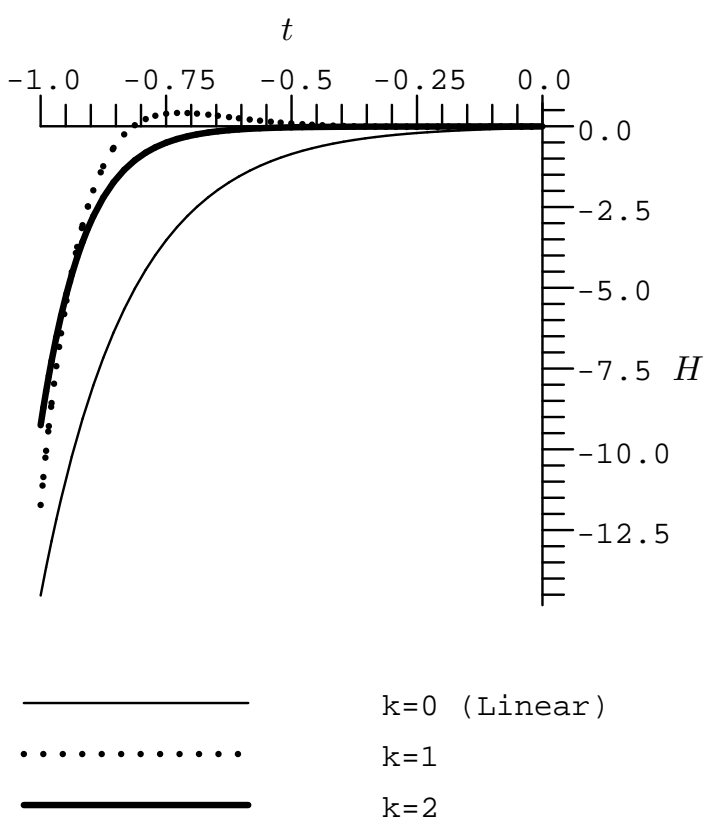

Fig. 7. Errors $H\left(x_{k}(t, \xi), p_{k}(t, \xi)\right)$

The family of approximations of the stable Lagrangian submanifold in Procedure 1

$$
\Lambda_{t}=\left\{(\boldsymbol{x}, \boldsymbol{p}) \mid \boldsymbol{p}=\frac{\partial \Phi}{\partial \boldsymbol{x}}(-t, \boldsymbol{x})^{T} e^{A^{T} t} \Gamma e^{A t} \Phi(-t, \boldsymbol{x})\right\}
$$

is calculated with

$$
A=\left(\begin{array}{cc}
0 & 1 \\
-1 & 0
\end{array}\right), \quad \Gamma=\left(\begin{array}{cc}
\sqrt{2 \sqrt{2}-1} \sqrt{2} & \sqrt{2}-1 \\
\sqrt{2}-1 & \sqrt{2 \sqrt{2}-1}
\end{array}\right)
$$

where $\Gamma$ is the stabilizing solution of

$$
\begin{gathered}
P A+A^{T} P-P R P+Q=0, \\
R=\left(\begin{array}{ll}
0 & 0 \\
0 & 1
\end{array}\right), \quad Q=I_{2}
\end{gathered}
$$

and we have written $\boldsymbol{x}=(x, \dot{x})^{T}, \boldsymbol{p}=\left(p_{1}, p_{2}\right)^{T}$.

For the calculation of $\Lambda_{t}$, it is necessary to differentiate the Jacobi elliptic functions with respect to the elliptic modulus, because $\Phi$ is differentiated by initial states $x, \dot{x}_{0}$ and $k$ in (32) is dependent on $x, \dot{x}_{0}$. We have listed some of the formulas required for this calculation in Appendix -C.

In Figures 8 and 9, approximations of $\partial V / \partial x$ and $\partial V / \partial \dot{x}$ with $t=-0.25$ are illustrated with the linear solution $\boldsymbol{p}=\Gamma \boldsymbol{x}$. The semi-transparent surfaces represent $\boldsymbol{p}=\Gamma \boldsymbol{x}$. It is seen that the approximate functions are tangent to the linear functions at the origin. 




Fig. 8. $\partial V / \partial x$ with the perturbation method and the first entry of the linear solution

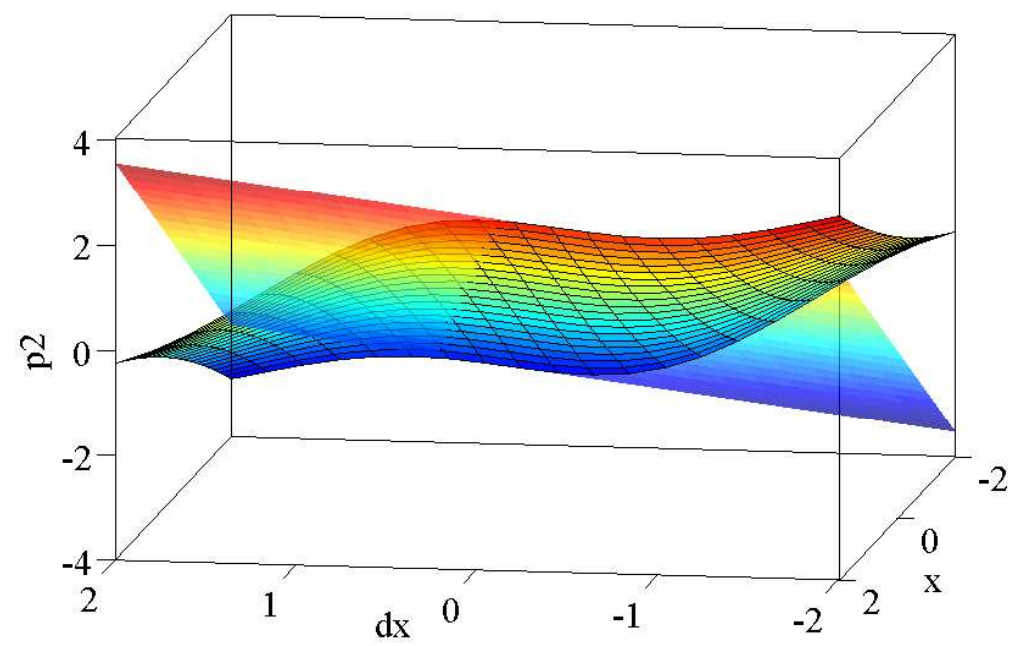

Fig. 9. $\partial V / \partial \dot{x}$ with the perturbation method and the second entry of the linear solution

B.2 The stable manifold theory approach

The associated Hamiltonian system to (31) is

$$
\begin{gathered}
\left(\begin{array}{l}
\dot{\boldsymbol{x}} \\
\dot{\boldsymbol{p}}
\end{array}\right)=\left(\begin{array}{cc}
A & -R \\
-Q & -A^{T}
\end{array}\right)\left(\begin{array}{l}
\boldsymbol{x} \\
\boldsymbol{p}
\end{array}\right)+\left(\begin{array}{c}
\bar{f}(\boldsymbol{x}, \boldsymbol{p}) \\
\bar{g}(\boldsymbol{x}, \boldsymbol{p})
\end{array}\right), \\
\bar{f}(\boldsymbol{x}, \boldsymbol{p})=\left(\begin{array}{c}
0 \\
-\varepsilon x^{3}
\end{array}\right), \quad \bar{g}(\boldsymbol{x}, \boldsymbol{p})=\left(\begin{array}{c}
3 \varepsilon p_{2} x^{2} \\
0
\end{array}\right) .
\end{gathered}
$$


The matrix $T$ that diagonalizes the linear part of $(35)$ is

$$
\left(\begin{array}{cccc}
1 & 0 & -\frac{\sqrt{2}+4}{4(2 \sqrt{2}+1) \sqrt{2 \sqrt{2}-1}} & 0 \\
0 & 1 & 0 & -\frac{1}{2 \sqrt{2 \sqrt{2}-1}} \\
\sqrt{2 \sqrt{2}-1} \sqrt{2} & \sqrt{2}-1 & 1 / 2 & -\frac{\sqrt{2}-1}{2 \sqrt{2 \sqrt{2}-1}} \\
\sqrt{2}-1 & \sqrt{2 \sqrt{2}-1} & -\frac{(\sqrt{2}-1)(\sqrt{2}+4)}{4(2 \sqrt{2}+1) \sqrt{2 \sqrt{2}-1}} & 1 / 2
\end{array}\right) .
$$

In the new cordinates $\left(\begin{array}{l}\boldsymbol{x}^{\prime} \\ \boldsymbol{p}^{\prime}\end{array}\right)=T\left(\begin{array}{c}\boldsymbol{x} \\ \boldsymbol{p}\end{array}\right),(35)$ is represented as

$$
\left(\begin{array}{c}
\dot{\boldsymbol{x}}^{\prime} \\
\dot{\boldsymbol{p}}^{\prime}
\end{array}\right)=\left(\begin{array}{cc}
A-R \Gamma & 0 \\
0 & -(A-R \Gamma)^{T}
\end{array}\right)\left(\begin{array}{l}
\boldsymbol{x}^{\prime} \\
\boldsymbol{p}^{\prime}
\end{array}\right)+\left(\begin{array}{c}
f\left(\boldsymbol{x}^{\prime}, \boldsymbol{p}^{\prime}\right) \\
g\left(\boldsymbol{x}^{\prime}, \boldsymbol{p}^{\prime}\right)
\end{array}\right)
$$

where $R, \Gamma$ are in $(33),(34)$ and $f, g$ are obtained, using $\left(\begin{array}{c}\boldsymbol{x} \\ \boldsymbol{p}\end{array}\right)=T^{-1}\left(\begin{array}{l}\boldsymbol{x}^{\prime} \\ \boldsymbol{p}^{\prime}\end{array}\right)$, as

$$
\left(\begin{array}{l}
f\left(\boldsymbol{x}^{\prime}, \boldsymbol{p}^{\prime}\right) \\
g\left(\boldsymbol{x}^{\prime}, \boldsymbol{p}^{\prime}\right)
\end{array}\right)=T\left(\begin{array}{l}
\bar{f}\left(\boldsymbol{x}\left(\boldsymbol{x}^{\prime}, \boldsymbol{p}^{\prime}\right), \boldsymbol{p}\left(\boldsymbol{x}^{\prime}, \boldsymbol{p}^{\prime}\right)\right) \\
\bar{g}\left(\boldsymbol{x}\left(\boldsymbol{x}^{\prime}, \boldsymbol{p}^{\prime}\right), \boldsymbol{p}\left(\boldsymbol{x}^{\prime}, \boldsymbol{p}^{\prime}\right)\right)
\end{array}\right) .
$$

Now, Procedure 2 can be applied to (36), and sequences (17) are transformed into the original coordinates with $\left(\begin{array}{l}\boldsymbol{x}^{\prime} \\ \boldsymbol{p}^{\prime}\end{array}\right)=T\left(\begin{array}{l}\boldsymbol{x} \\ \boldsymbol{p}\end{array}\right)$. Fig. 10 shows the second-order approximation $(k=2)$ of $\partial V / \partial \dot{x}$ and the second entry of the linear solution (semi-transparent surface). Also, Fig. 11 shows the surfaces representing $\partial V / \partial \dot{x}$ with the perturbation and stable manifold $(k=2)$ methods to compare the two methods. The semi-transparent surface corresponds to the one with the perturbation method (the same surface in Fig. 9). Figures 9-11 are drawn from the same directions with the same scales to compare the surfaces. Since the optimal feedback law of this problem does not require $\partial V / \partial x$, the surface for this derivative is not presented.

\section{CONCLUDING REMARKS}

In this paper, we proposed two analytical approximation approaches for obtaining the stabilizing solution of the Hamilton-Jacobi equation using a Hamiltonian perturbation technique and stable manifold theory. The proposed methods give approximated flows on the stable Lagrangian submanifold of the associated Hamiltonian system as functions of time and initial states. The perturbation approach provides a set of approximations for the derivative of the stabilizing solution. On the other hand, in the stable manifold approach, parametrizations of the stable Lagrangian submanifold are given. Since these methods produce analytical expressions for approximations, it is possible to compute the solution of the Hamilton-Jacobi equation using its integral expressions (§VI-B). Moreover, in the case of polynomial nonlinear systems, each approximation step yields the Hamiltonian flows with exponential and trigonometric functions in the stable manifold method, providing approximate solutions as polynomial functions ( $\S \mathrm{VI}-\mathrm{C})$. In this case, the calculations are all algebraic, that is, no numerical integration is required and no equations need to be solved. Since these methods focus on the stable manifold of the Hamiltonian system, the closed loop system stability is guaranteed and can be enhanced by taking higher order approximations. A one-dimensional example shows that they are effective in that the optimal feedback is well approximated and that, compared to the Taylor expansion method, they give better results especially further from the equilibrium. An example of a nonlinear spring-mass system is illustrated to show how they work for a higher dimensional system.

Acknowledgement. The first author was supported by the Scientist Exchange Program between the Japan Society for the Promotion of Science (JSPS) and the Netherlands Organisation for Scientific Research (NWO). 


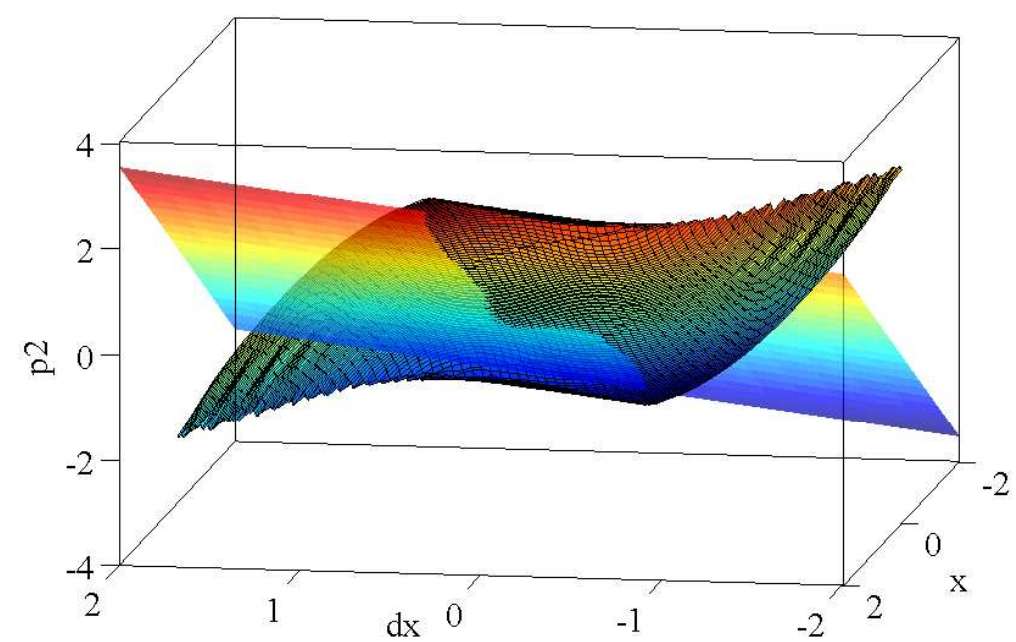

Fig. 10. $\partial V / \partial \dot{x}$ with the stable manifold method and the second entry of the linear solution

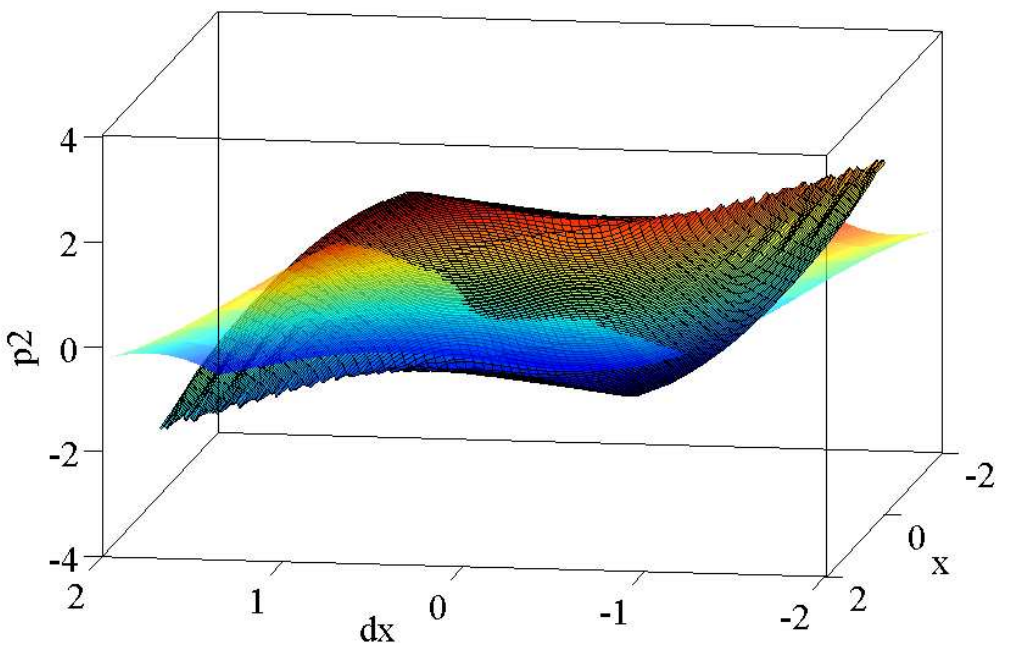

Fig. 11. $\partial V / \partial \dot{x}$ with the perturbation and stable manifold methods

\section{REFERENCES}

[1] R. Abraham and J. E. Marsden. Foundations of Mechanics. Addison-Wesley, New York, 2nd edition, 1979.

[2] M. Abramowitz and I. A. Stegun. Handbook of Mathematical Functions : With Formulas, Graphs, and Mathematical Tables. New York : Dover, 1965.

[3] S. Arimoto. Optimal feedback control minimizing the effects of noise disturbance. Trans. SICE, 2(1):1-7, 1966. (in Japanese).

[4] V. I. Arnold. Mathematical Methods of Classical Mechanics. Springer-Verlag, 2nd edition, 1989.

[5] J. A. Ball, J. W. Helton, and M. L. Walker. $H_{\infty}$ control for nonlinear systems with output feedback. IEEE Trans. Automat. Control, 38(4):546-559, 1993.

[6] J. A. Ball, M. A. Petersen, and A. van der Schaft. Inner-outer factorization for nonlinear noninvertible systems. IEEE Trans. Automat. Control, 49(4):483-492, 2004.

[7] J. A. Ball and A. J. van der Schaft. J-inner-outer factorization, J-spectral factorization, and robust control for nonlinear systems. IEEE Trans. Automat. Control, 41(3):379-392, 1996.

[8] M. Bardi and I. Capuzzo-Dolcetta. Optimal Control and Viscosity Solutions of Hamilton-Jacobi-Bellman Equations. Birkhäuser, 1997.

[9] R. W. Beard, G. N. Sardis, and J. T. Wen. Galerkin approximations of the generalized Hamilton-Jacobi-Bellman equation. Automatica, 33(12):2195-2177, 1997.

[10] S.-N. Chow and J. K. Hale. Methods of Bifurcation Theory. Springer-Verlag, 1982.

[11] M. G. Crandall and P. L. Lions. Viscosity solutions of Hamilton-Jacobi equations. Trans. AMS, 277:1-43, 1983.

[12] P. E. Crouch and A. J. van der Schaft. Variational and Hamiltonian Control Systems. Springer-Verlag, 1987.

[13] W. H. Fleming and H. M. Soner. Controlled Markov Processes and Viscosity Solutions. Springer, 2nd edition, 2005.

[14] B. A. Francis. A Course in $H_{\infty}$ Control Theory. Springer, 1986. 
[15] K. Fujimoto and J. M. A. Scherpen. Nonlinear input-normal realizations based on the differential eigenstructure of Hankel operators. IEEE Trans. Automat. Control, 50(1):2-18, 2005.

[16] W. L. Garrard. Additional results on sub-optimal feedback control of non-linear systems. Int. J. Control, 10(6):657-663, 1969.

[17] W. L. Garrard and J. M. Jordan. Design of nonlinear automatic flight control systems. Automatica, 13(5):497-505, 1977.

[18] H. Goldstein. Classical Mechanics. Addison-Wesley, 3rd edition, 2001.

[19] D. Hill and P. Moylan. The stability of nonlinear dissipative systems. IEEE Trans. Automat. Control, 21(10):708-711, 1976.

[20] Y. Huang and W.-M. Lu. Nonlinear optimal control: Alternatives to Hamilton-Jacobi equation. In Proc. of IEEE Conference on Decision and Control, pages 3942-3947, 1996.

[21] A. Isidori and A. Astolfi. Disturbance attenuation and $H_{\infty}$-control via measurement feedback in nonlinear systems. IEEE Trans. Automat. Control, 37(9):1283-1293, 1992.

[22] B. Krauskopf, H. M. Osinga, E. J. Doedel, M. E. Henderson, J. Guckenheimer, A. Vladimirsky, M. Dellnitz, and O. Junge. A survey of methods for computing (un)stable manifolds of vector fields. International Journal of Bifurcation and Chaos, 15(3):763-791, 2005.

[23] P. Lancaster and L. Rodman. Algebraic Riccati Equations. Oxford University Press, 1995.

[24] R. J. Leake and R.-W. Liu. Construction of suboptimal control sequences. SIAM J. Control Optim., 5(1):54-63, 1967.

[25] E. B. Lee and L. Markus. Foundations of Optimal Control Theory. John Wiley, New York, 1967.

[26] P. Libermann and C.-M. Marle. Symplectic Geometry and Analytical Mechanics. D. Reidel, 1987.

[27] D. L. Lukes. Optimal regulation of nonlinear dynamical systems. SIAM J. Control Optim., 7(1):75-100, 1969.

[28] J. Markman and I. N. Katz. An iterative algorithm for solving Hamilton-Jacobi type equations. SIAM Journal on Scientific Computing, 22(1):312-329, 2000.

[29] C. P. Mracek and J. R. Cloutier. Control designs for the nonlinear benchmark problem via the state-dependent Riccati equation method. Int. J. Robust and Nonlinear Control, 8:401-433, 1998.

[30] Y. Nishikawa, N. Sannomiya, and H. Itakura. A method for suboptimal design of nonlinear feedback systems. Automatica, 7:703-712, 1971.

[31] T. Oshima and H. Komatsu. Partial Differential Equations of the First Order. Iwanami Shoten, Tokyo, 1977. (in Japanese).

[32] H. M. Osinga and J. Hauser. The geometry of the solution set of nonlinear optimal control problems. Journal of Dynamics and Differential Equations, 18(4):881-900, 2006.

[33] J. E. Potter. Matrix quadratic solutions. SIAM J. Appl. Math., 14:496-501, 1966.

[34] C. Robinson. Dynamical Systems: Stability, Symbolic Dynamics, and Chaos. CRC Press, 2nd edition, 1998.

[35] N. Sakamoto. Analysis of the Hamilton-Jacobi equation in nonlinear control theory by symplectic geometry. SIAM J. Control Optim., 40(6):1924-1937, 2002.

[36] J. M. A. Scherpen. Balancing for nonlinear systems. Syst. Control Lett., 21(2):143-153, 1993.

[37] P. Soravia. $H^{\infty}$ control of nonlinear systems: Differential games and viscosity solutions. SIAM J. Control Optim., 34(3):10711097, 1996.

[38] A. J. van der Schaft. On a state space approach to nonlinear $H_{\infty}$ control. Syst. Control Lett., 16(1):1-18, 1991.

[39] A. J. van der Schaft. $L_{2}$-gain analysis of nonlinear systems and nonlinear state feedback $H_{\infty}$ control. IEEE Trans. Automat. Control, 37(6):770-784, 1992.

[40] A. J. van der Schaft. $L_{2}$-Gain and Passivity Techniques in Nonlinear Control. Springer, 2nd edition, 1999.

[41] A. Wernrud and A. Rantzer. On approximate policy iteration for continuous-time systems. In Proc. of IEEE Conference on Decision and Control and European Control Conference, 2005.

[42] J. C. Willems. Dissipative dynamical systems-Part I, II. Arch. Rational Mechanics and Analysis, 45:321-393, 1972.

\section{APPENDIX}

\section{A. The variation of constants technique in Hamiltonian perturbation theory}

We review, in this section, one of the Hamiltonian perturbation techniques which is a simple consequence of the Hamilton-Jacobi theory (see, e.g., [4], [18]).

Let

$$
H(x, p)=H_{0}(x, p)+H_{1}(x, p)
$$

be the Hamiltonian with the integrable part $H_{0}$ and the perturbation $H_{1}$. By the integrability condition, the Hamilton-Jacobi equation

$$
H_{0}\left(x, \frac{\partial S}{\partial x}\right)+\frac{\partial S}{\partial t}=0
$$

has a complete solution $S\left(x_{1}, \ldots, x_{n}, t, P_{1}, \ldots, P_{n}\right)$, where $P_{1}, \ldots, P_{n}$ are arbitrary constants. By the canonical coordinate transformation $(x, p) \rightarrow(X, P)$ defined by

$$
p_{j}=\frac{\partial S}{\partial x_{j}}, \quad X_{j}=\frac{\partial S}{\partial P_{j}}
$$


the unperturbed Hamiltonain in the coordinates $(X, P)$ becomes 0 and the unperturbed equations of motion

$$
\dot{x}_{j}=\frac{\partial H_{0}}{\partial p_{j}}, \quad \dot{p}_{j}=-\frac{\partial H_{0}}{\partial x_{j}}
$$

are converted into

$$
\dot{X}_{j}=0, \quad \dot{P}_{j}=0
$$

By the canonical transformation (38), the new Hamiltonian for the perturbed equations of motion is $H_{1}(x, p)$ since by $(37) S$ satisfies

$$
H\left(x, \frac{\partial S}{\partial x}\right)+\frac{\partial S}{\partial t}=H_{1}(x, p)
$$

Therefore,

$$
\dot{x}_{j}=\frac{\partial H}{\partial p_{j}}, \quad \dot{p}_{j}=-\frac{\partial H}{\partial x_{j}}
$$

are converted into

$$
\dot{X}_{j}=\frac{\partial H_{1}}{\partial P_{j}}(x, p) \quad \dot{P}_{j}=-\frac{\partial H_{1}}{\partial X_{j}}(x, p)
$$

where, from (38), $x_{j}=x_{j}(t, X, P)$ and $p_{j}=p_{j}(t, X, P)$.

\section{B. Proof of Theorem 5}

From Assumptions 3 and 4, the following inequalities are derived. (In this section, we leave out the dependence of $x_{k}$ and $y_{k}$ on $\xi$ for the sake of simplicity.)

- If $|x|+|y| \leqslant L_{1}$, then

$$
\begin{aligned}
|f(t, x, y)| & \leqslant \delta_{1}(|x|+|y|)(|x|+|y|) \\
& \leqslant M_{1}(|x|+|y|)^{2} .
\end{aligned}
$$

If $|x|+|y| \leqslant L_{2}$, then

$$
\begin{aligned}
|g(t, x, y)| & \leqslant \delta_{2}(|x|+|y|)(|x|+|y|) \\
& \leqslant M_{2}(|x|+|y|)^{2} .
\end{aligned}
$$

- If $|x|,\left|x^{\prime}\right| \leqslant \bar{x}$ and $|y|,\left|y^{\prime}\right| \leqslant \bar{y}$ for some positive constants $\bar{x}, \bar{y}$ satisfying $\bar{x}+\bar{y} \leqslant L_{1}$, then

$$
\begin{aligned}
\mid f(t, x, y) & -f\left(t, x^{\prime}, y^{\prime}\right) \mid \\
& \leqslant \delta_{1}(\bar{x}+\bar{y})\left(\left|x-x^{\prime}\right|+\left|y-y^{\prime}\right|\right) \\
& \leqslant M_{1}(\bar{x}+\bar{y})\left(\left|x-x^{\prime}\right|+\left|y-y^{\prime}\right|\right) .
\end{aligned}
$$

If $|x|,\left|x^{\prime}\right| \leqslant \bar{x}$ and $|y|,\left|y^{\prime}\right| \leqslant \bar{y}$ for some positive constants $\bar{x}, \bar{y}$ satisfying $\bar{x}+\bar{y} \leqslant L_{2}$, then

$$
\begin{aligned}
\mid g(t, x, y) & -g\left(t, x^{\prime}, y^{\prime}\right) \mid \\
& \leqslant \delta_{1}(\bar{x}+\bar{y})\left(\left|x-x^{\prime}\right|+\left|y-y^{\prime}\right|\right)
\end{aligned}
$$




$$
\leqslant M_{2}(\bar{x}+\bar{y})\left(\left|x-x^{\prime}\right|+\left|y-y^{\prime}\right|\right)
$$

(i) First, we show that the limits of sequences (17) and (18) satisfy (16). By taking limit in (17), we have the integral equations for $x(t)$ and $y(t)$

$$
\begin{aligned}
& x(t)=e^{F t} \xi+\int_{0}^{t} e^{F(t-s)} f(s, x(s), y(s)) d s \\
& y(t)=-\int_{t}^{\infty} e^{-F^{T}(t-s)} g(s, x(s), y(s)) d s
\end{aligned}
$$

from which one can see that $x(t)$ and $y(t)$ satisfy (16).

(ii) For each $k=0,1,2, \ldots, x_{k}(t)$ and $y_{k}(t)$ have the following estimates;

$$
\left|x_{k}(t)\right| \leqslant \alpha_{k} e^{-b t}, \quad\left|y_{k}(t)\right| \leqslant \beta_{k} e^{-2 b t},
$$

where $\alpha_{k}$ and $\beta_{k}$ are the constants defined by

$$
\left\{\begin{array}{l}
\alpha_{k+1}=\frac{2 a M_{1}}{b}\left(\alpha_{k}^{2}+\beta_{k}^{2}\right)+a|\xi| \\
\beta_{k+1}=\frac{2 a M_{2}}{3 b}\left(\alpha_{k}^{2}+\beta_{k}^{2}\right) \\
\alpha_{0}=a|\xi|, \quad \beta_{0}=0 .
\end{array}\right.
$$

Indeed, $\left|x_{0}(t)\right| \leqslant a|\xi| e^{-b t}$ from Assumption 3 and $y_{0}(t)=0$ from which the claim for $n=0$ follows. Let us assume that the claim holds for $k$.

$$
\begin{aligned}
& \left|x_{k+1}(t)\right| \\
& \leqslant a|\xi| e^{-b t}+\int_{0}^{t} a e^{-b(t-s)}\left|f\left(s, x_{k}(t), y_{k}(s)\right)\right| d s \\
& \leqslant a|\xi| e^{-b t}+a M_{1} e^{-b t} \int_{0}^{t} e^{b s}\left(\left|x_{k}(s)+y_{k}(s)\right|\right)^{2} d s \\
& \leqslant a|\xi| e^{-b t}+2 a M_{1} e^{-b t} \int_{0}^{t} e^{b s}\left(\alpha_{k}^{2} e^{-2 b s}+\beta_{k}^{2} e^{-4 b s}\right) d s \\
& \leqslant a|\xi| e^{-b t}+2 a M_{1}\left(\alpha_{k}^{2}+\beta_{k}^{2}\right) e^{-b t} \int_{0}^{t} e^{-b s} d s \\
& \leqslant a|\xi| e^{-b t}+\frac{2 a M_{1}}{b}\left(\alpha_{k}^{2}+\beta_{k}^{2}\right) e^{-b t} \\
& =\left\{\frac{2 a M_{1}}{b}\left(\alpha_{k}{ }^{2}+\beta_{k}{ }^{2}\right)+a|\xi|\right\} e^{-b t},
\end{aligned}
$$

where we have used (39) and (40). Similar calculations give

$$
\begin{aligned}
\left|y_{k+1}(t)\right| & \leqslant \int_{t}^{\infty} a e^{-b(s-t)}\left|g\left(s, x_{k}(s), y_{k}(s)\right)\right| d s \\
& \leqslant \frac{2 a M_{2}}{3 b}\left(\alpha_{k}^{2}+\beta_{k}^{2}\right) e^{-2 b t} .
\end{aligned}
$$

Thus, (43) for $k+1$ holds with $\alpha_{k+1}$ and $\beta_{k+1}$ in (44).

(iii) Next, we show that for sufficiently small $|\xi|,\left\{\alpha_{k}\right\}$ and $\left\{\beta_{k}\right\}$ are bounded and monotonically increasing sequences and therefore, $\lim _{k \rightarrow \infty} \alpha_{k}=: \underline{\alpha}, \lim _{k \rightarrow \infty} \beta_{k}=: \underline{\beta}$ exists. Furthermore, we 
show that $\underline{\alpha}, \underline{\beta} \rightarrow 0$ when $|\xi| \rightarrow 0$. Let $=2 a M_{1} / b, c_{2}=a|\xi|\left(=\alpha_{0}\right)$, and $c_{3}=2 a M_{2} /(3 b)$. Then, it follows that

$$
\begin{aligned}
\alpha_{k+1} & -\alpha_{k} \\
& =c_{1}\left\{\left(\alpha_{k}+\alpha_{k-1}\right)\left(\alpha_{k}-\alpha_{k-1}\right)+\left(\beta_{k}+\beta_{k+1}\right)\left(\beta_{k}-\beta_{k-1}\right)\right\} \\
\beta_{k+1} & -\beta_{k} \\
& =c_{3}\left\{\left(\alpha_{k}+\alpha_{k-1}\right)\left(\alpha_{k}-\alpha_{k-1}\right)+\left(\beta_{k}+\beta_{k+1}\right)\left(\beta_{k}-\beta_{k-1}\right)\right\},
\end{aligned}
$$

therefore, $\alpha_{k+1}>\alpha_{k}$ and $\beta_{k+1}>\beta_{k}$ if $\alpha_{1}>\alpha_{0}$ and $\beta_{1}>\beta_{0}$, which is readily verified. We next claim that the equation

$$
\left\{\begin{array}{l}
\alpha=c_{1}\left(\alpha^{2}+\beta^{2}\right)+c_{2} \\
\beta=c_{3}\left(\alpha^{2}+\beta^{2}\right)
\end{array}\right.
$$

has a solution for sufficiently small $|\xi|$. To prove the claim, define a map $T: \mathbb{R}^{2} \rightarrow \mathbb{R}^{2}$ by

$$
T(\alpha, \beta)=\left(\begin{array}{c}
c_{1}\left(\alpha^{2}+\beta^{2}\right)+c_{2} \\
c_{3}\left(\alpha^{2}+\beta^{2}\right)
\end{array}\right)
$$

Since

$$
\begin{aligned}
& \left|T(\alpha, \beta)-T\left(\alpha^{\prime}, \beta^{\prime}\right)\right| \\
& \quad \leqslant\left(c_{1}+c_{3}\right)\left\{\left(\alpha+\alpha^{\prime}\right)\left|\alpha-\alpha^{\prime}\right|+\left(\beta+\beta^{\prime}\right)\left|\beta-\beta^{\prime}\right|\right\}
\end{aligned}
$$

it follows that for small $|\xi|, T(U) \subset U$ and $T: U \rightarrow U$ is a contraction map in a neighborhood $U$ of $(0,0)$. Therefore, $T$ has the unique fixed point $(\underline{\alpha}, \underline{\beta})$ in $U$, which is a solution of (45). We note that when $|\xi| \neq 0, \underline{\alpha}>0$ and $\underline{\beta}>0$. It can be shown, in the same way as the monotonicity proof of $\left\{\alpha_{k}\right\}$ and $\left\{\beta_{k}\right\}$, that $\alpha_{k} \leqslant \underline{\alpha}$ and $\beta_{k} \leqslant \underline{\beta}$ as long as $\alpha_{0} \leqslant \underline{\alpha}$ and $\beta_{0} \leqslant \underline{\beta}$, which is obvious from (44) and (45). Thus, we have shown that $\left\{\alpha_{k}\right\}$ and $\left\{\beta_{k}\right\}$ are bounded. Therefore, their limits exist and coincide with $(\underline{\alpha}, \underline{\beta})$ since there is no other solution of $(45)$ in $U$. Because $(\underline{\alpha}, \underline{\beta})$ is the solution of $(45)$, it is clear that $(\underline{\alpha}, \underline{\beta}) \rightarrow(0,0)$ as $|\xi| \rightarrow 0$.

(iv) Next, we show that

$$
\begin{gathered}
\left|x_{k}(t)-x_{k+1}(t)\right| \leqslant \gamma_{k} e^{-b t} \\
\left|y_{k}(t)-y_{k+1}(t)\right| \leqslant \varepsilon_{k} e^{-2 b t}
\end{gathered}
$$

where $\left\{\gamma_{k}\right\},\{\varepsilon\}$ are the positive sequences defined by

$$
\left\{\begin{array}{l}
\gamma_{k+1}=\frac{a(\underline{\alpha}+\underline{\beta}) M_{1}}{b}\left(\gamma_{k}+\varepsilon_{k}\right) \\
\varepsilon_{k+1}=\frac{a(\underline{\alpha}+\underline{\beta}) M_{2}}{3 b}\left(\gamma_{k}+\varepsilon_{k}\right) \\
\gamma_{1}=\frac{a^{3} M_{1}|\xi|^{2}}{b}, \quad \varepsilon_{1}=\frac{a^{3} M_{2}|\xi|^{2}}{3 b} .
\end{array}\right.
$$

Indeed, for $k=1$, using (41) and (42), we have

$$
\begin{aligned}
\mid x_{1}(t) & -x_{0}(t) \mid \\
& \leqslant \int_{0}^{t} a e^{-b(t-s)}\left|f\left(s, x_{0}(s), y_{0}(s)\right)\right| d s
\end{aligned}
$$




$$
\begin{aligned}
& \leqslant a M_{1} e^{-b t} \int_{0}^{t} e^{b s}\left(\left|x_{0}(s)\right|+\left|y_{0}(s)\right|\right)^{2} d s \\
& \leqslant a^{3} M_{1}|\xi|^{2} e^{-b t} \int_{0}^{t} e^{-b s} d s \\
& \leqslant \frac{a^{3} M_{1}|\xi|^{2}}{b} e^{-b t}, \\
\mid y_{1}(t) & -y_{0}(t) \mid \\
& \leqslant \int_{t}^{\infty} a e^{-b(s-t)}\left|g\left(s, x_{0}(s), y_{0}(s)\right)\right| d s \\
& \leqslant a e^{b t} \int_{t}^{\infty} e^{-b s} M_{2}\left|x_{0}(s)\right|^{2} d s \\
& \leqslant a^{3} M_{2}|\xi|^{2} e^{b t} \int_{t}^{\infty} e^{-3 b s} d s \\
& =\frac{a^{3} M_{2}|\xi|^{2}}{3 b} e^{-2 b t} .
\end{aligned}
$$

Let us assume (46) and (47) for $k$. For $k+1$, using (41) and the monotonicity of $\delta_{1}$

$$
\begin{aligned}
& \mid \begin{array}{l}
x_{k+1}(t)-x_{k}(t) \mid \\
\leqslant \int_{0}^{t} a e^{-b(t-s)}\left|f\left(s, x_{k}(s), y_{k}(s)\right)-f\left(s, x_{k-1}(s), y_{k-1}(s)\right)\right| d s \\
\leqslant a e^{-b t} \int_{0}^{t} e^{b s} \delta_{1}\left(\underline{\alpha} e^{-b s}+\underline{\beta} e^{-2 b s}\right) \\
\quad \times\left(\left|x_{k}-x_{k-1}\right|+\left|y_{k}-y_{k-1}\right|\right) d s \\
\leqslant a(\underline{\alpha}+\underline{\beta}) M_{1} e^{-b t} \int_{0}^{t}\left(\left|x_{k}-x_{k-1}\right|+\left|y_{k}-y_{k-1}\right|\right) d s \\
\leqslant a(\underline{\alpha}+\underline{\beta}) M_{1} e^{-b t} \int_{0}^{t}\left(\gamma_{k} e^{-b s}+\varepsilon_{k} e^{-2 b s}\right) d s \\
\leqslant a(\underline{\alpha}+\underline{\beta}) M_{1}\left(\gamma_{k}+\varepsilon_{k}\right) e^{-b t} \int_{0}^{t} e^{-b s} d s \\
\leqslant \frac{a(\underline{\alpha}+\underline{\beta}) M_{1}}{b}\left(\gamma_{k}+\varepsilon_{k}\right) e^{-b t},
\end{array}
\end{aligned}
$$

and using (42) and the monotonicity of $\delta_{2}$

$$
\begin{aligned}
& \left|y_{k+1}(t)-y_{k}(t)\right| \\
& \leqslant \int_{t}^{\infty} a e^{-b(s-t)}\left|g\left(s, x_{k}(s), y_{k}(s)\right)-g\left(s, x_{k-1}(s), y_{k-1}(s)\right)\right| d s \\
& \leqslant a(\underline{\alpha}+\underline{\beta}) M_{2} e^{b t} \int_{t}^{\infty} e^{-2 b s}\left(\left|x_{k}-x_{k-1}\right|+\left|y_{k}-y_{k-1}\right|\right) d s \\
& \leqslant \frac{a(\underline{\alpha}+\underline{\beta}) M_{2}}{3 b}\left(\gamma_{k}+\varepsilon_{k}\right) e^{-2 b t} .
\end{aligned}
$$

(v) Lastly, we prove that for sufficiently small $|\xi|,\left\{\gamma_{k}\right\}$ and $\left\{\varepsilon_{k}\right\}$ are monotonically decreasing sequences and $\lim _{t \rightarrow \infty} \gamma_{k}=\lim _{t \rightarrow \infty} \varepsilon_{k}=0$. As a matter of fact, it can be easily seen, from the 
definition of the sequences, that $\gamma_{k}>\gamma_{k+1}$ and $\varepsilon_{k}>\varepsilon_{k+1}$ for all $k=1,2, \ldots$ if $\gamma_{1}>\gamma_{2}$ and $\varepsilon_{1}>\varepsilon_{2}$. However, these can be verified from

$$
\begin{aligned}
& \gamma_{1}-\gamma_{2}=\left\{\left(1-\frac{a(\underline{\alpha}+\underline{\beta}) M_{1}}{b}\right) \frac{a^{3} M_{1}}{b}-\frac{a(\underline{\alpha}+\underline{\beta}) M_{1}}{b} \frac{a^{3} M_{2}}{3 b}\right\}|\xi|^{2} \\
& \varepsilon_{1}-\varepsilon_{2}=\left\{\left(1-\frac{a(\underline{\alpha}+\underline{\beta}) M_{2}}{3 b}\right) \frac{a^{3} M_{1}}{b}-\frac{a(\underline{\alpha}+\underline{\beta}) M_{2}}{3 b} \frac{a^{3} M_{2}}{3 b}\right\}|\xi|^{2},
\end{aligned}
$$

and from the fact that $\underline{\alpha}, \underline{\beta} \rightarrow 0$ as $|\xi| \rightarrow 0$. Therefore, the limits $\lim _{k \rightarrow \infty} \gamma_{k}, \lim _{k \rightarrow \infty} \varepsilon_{k}$ exist and coincide with the solution of

$$
\left\{\begin{array}{l}
\gamma=\frac{a(\underline{\alpha}+\underline{\beta}) M_{1}}{b}(\gamma+\varepsilon) \\
\varepsilon=\frac{a(\underline{\alpha}+\underline{\beta}) M_{2}}{3 b}(\gamma+\varepsilon),
\end{array}\right.
$$

which has the unique solution $(0,0)$.

C. The Jacobi elliptic functions[2]

C.1 Derivation of (32)

Let $x^{2}=a^{2}$ be the solution of $2 E-x^{2}-(\varepsilon / 2) x^{4}=0$. Then, from $E$ (constant) $=\frac{1}{2} x_{0}^{2}+\frac{1}{4} \varepsilon x_{0}^{4}+\frac{1}{2} \dot{x}_{0}^{2}$,

$$
\begin{aligned}
t & = \pm \int \frac{d x}{\sqrt{2 E-x^{2}-(\varepsilon / 2) x^{4}}} \\
& = \pm \int \frac{d x}{\sqrt{(\varepsilon / 2)\left(a^{2}-x^{2}\right)\left(x^{2}+a^{2}+\varepsilon / 2\right)}} \\
& =\frac{ \pm 1}{\sqrt{1+\varepsilon a^{2}}} \int_{0}^{\varphi} \frac{d \theta}{\sqrt{1-k^{2} \sin ^{2} \theta}}+t_{0} \\
& =\frac{ \pm 1}{\sqrt{1+\varepsilon a^{2}}} \operatorname{am}^{-1}(\varphi, k)+t_{0},
\end{aligned}
$$

where we have set $x=a \cos \theta, k=\sqrt{\frac{\varepsilon a^{2}}{2\left(1+\varepsilon a^{2}\right)}}$ and am is Jacobi's amplitude function. Thus, we get (32) from $\operatorname{cn}(x, k)=\cos (\operatorname{am}(x, k))$.

C.2 Formulas

Differentiations with respect to $x$ :

$$
\begin{gathered}
\frac{\partial \operatorname{sn}(x, k)}{\partial x}=\operatorname{cn}(x, k) \operatorname{dn}(x, k), \\
\frac{\partial \operatorname{cn}(x, k)}{\partial x}=-\operatorname{sn}(x, k) \operatorname{dn}(x, k), \\
\frac{\partial \operatorname{dn}(x, k)}{\partial x}=-k^{2} \operatorname{sn}(x, k) \operatorname{cn}(x, k) .
\end{gathered}
$$

Addition formulas:

$$
\begin{aligned}
& \operatorname{sn}(x+y, k) \\
& =\frac{\operatorname{sn}(x, k) \operatorname{cn}(y, k) \operatorname{dn}(y, k)+\operatorname{sn}(y, k) \operatorname{cn}(x, k) \operatorname{dn}(x, k)}{1-k^{2} \operatorname{sn}^{2}(x, k) \operatorname{sn}^{2}(y, k)} \\
& \operatorname{cn}(x+y, k)
\end{aligned}
$$




$$
\begin{aligned}
& =\frac{\operatorname{cn}(x, k) \operatorname{cn}(y, k)-\operatorname{sn}(x, k) \operatorname{sn}(y, k) \operatorname{dn}(x, k) \operatorname{dn}(y, k)}{1-k^{2} \operatorname{sn}^{2}(x, k) \operatorname{sn}^{2}(y, k)}, \\
& \operatorname{dn}(x+y, k) \\
& =\frac{\operatorname{dn}(x, k) \operatorname{dn}(y, k)-k^{2} \operatorname{sn}(x, k) \operatorname{sn}(y, k) \operatorname{cn}(x, k) \operatorname{cn}(y, k)}{1-k^{2} \operatorname{sn}^{2}(x, k) \operatorname{sn}^{2}(y, k)} .
\end{aligned}
$$

Differentiation with respect to the elliptic modulus $k$ :

$$
\begin{aligned}
\frac{\partial \operatorname{sn}(x, k)}{\partial k}= & \frac{k\left(\operatorname{sn}(x, k)-\operatorname{sn}^{3}(x, k)\right)}{1-k^{2}}+\frac{x \operatorname{cn}(x, k) \operatorname{dn}(x, k)}{k} \\
& -\frac{\operatorname{cn}(x, k) \operatorname{dn}(x, k)}{k\left(1-k^{2}\right)}\left(\operatorname{zn}(x, k)+\frac{x E(x, k)}{K(k)}\right) \\
\frac{\partial \operatorname{cn}(x, k)}{\partial k}= & -\frac{k \operatorname{sn}^{2}(x, k) \operatorname{cn}(x, k)}{1-k^{2}}-\frac{\operatorname{dn}(x, k) \operatorname{sn}(x, k) x}{k} \\
& +\frac{\operatorname{dn}(x, k) \operatorname{sn}(x, k)}{k\left(1-k^{2}\right)}\left(\operatorname{zn}(x, k)+\frac{x E(k)}{K(k)}\right) \\
\frac{\partial \operatorname{dn}(x, k)}{\partial k}= & -k \operatorname{cn}(x, k) \operatorname{sn}(x, k) x-\frac{k \operatorname{sn}^{2}(x, k) \operatorname{dn}(x, k)}{1-k^{2}} \\
& +\frac{k \operatorname{cn}(x, k) \operatorname{sn}(x, k)}{1-k^{2}}\left(\operatorname{zn}(x, k)+\frac{x E(k)}{K(k)}\right)
\end{aligned}
$$

where $K(k)$ and $E(k)$ are the complete elliptic integrals of the first and second kind, respectively, defined by

$$
\begin{gathered}
K(k)=\int_{0}^{1} \frac{d t}{\sqrt{\left(1-t^{2}\right)\left(1-k^{2} t^{2}\right)}}, \\
E(k)=\int_{0}^{1} \frac{\sqrt{1-k^{2} t^{2}}}{\sqrt{1-t^{2}}} d t
\end{gathered}
$$

and zn is Jacobi's zeta function defined by

$$
\operatorname{zn}(x, k)=\int_{0}^{x} \operatorname{dn}^{2}(t, k) d t-\frac{E(k)}{K(k)} x .
$$

